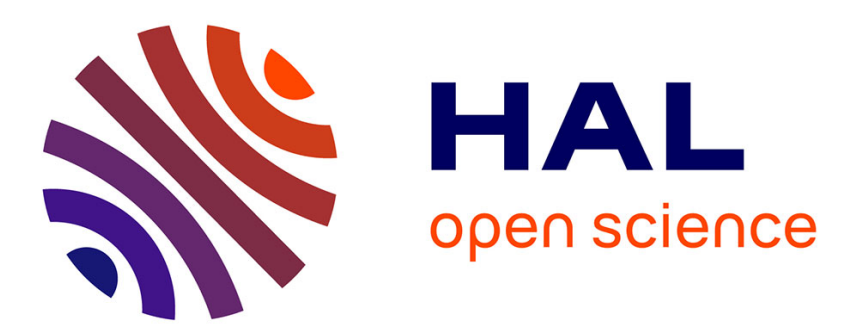

\title{
Free vibration analysis of plates taking into account rotary inertia and shear deformation via three alternative theories: a Lévy-type solution
}

\author{
F. Hache, I. Elishakoff, Noël Challamel
}

\section{- To cite this version:}

F. Hache, I. Elishakoff, Noël Challamel. Free vibration analysis of plates taking into account rotary inertia and shear deformation via three alternative theories: a Lévy-type solution. Acta Mechanica, 2017, 228 (10), pp.3633-3655. 10.1007/s00707-017-1890-8 . hal-01693900

\author{
HAL Id: hal-01693900 \\ https://hal.science/hal-01693900
}

Submitted on 31 Dec 2019

HAL is a multi-disciplinary open access archive for the deposit and dissemination of scientific research documents, whether they are published or not. The documents may come from teaching and research institutions in France or abroad, or from public or private research centers.
L'archive ouverte pluridisciplinaire HAL, est destinée au dépôt et à la diffusion de documents scientifiques de niveau recherche, publiés ou non, émanant des établissements d'enseignement et de recherche français ou étrangers, des laboratoires publics ou privés. 
F. Hache · I. Elishakoff $\cdot$ N. Challamel

\title{
Free vibration analysis of plates taking into account rotary inertia and shear deformation via three alternative theories: a Lévy-type solution
}

\begin{abstract}
This paper deals with the exact calculation of natural frequencies of a plate with two opposite edges simply supported considering three versions of the Uflyand-Mindlin plate theory: the original UflyandMindlin plate theory, the truncated version of this model as suggested by Elishakoff, and the recently proposed Uflyand-Mindlin plate theory based on slope inertia. The comparison between the frequencies using the different models and with those found in the literature using numerical methods shows the efficiency of the models and the methods presented hereinafter.
\end{abstract}

\section{Introduction}

Rectangular plates are widely used in engineering practice. It appears crucial to understand their free vibrational behavior. Different models exist to describe the mechanical behavior of plates. The Kirchhoff-Love (KL) plate theory [1], also referred to as the classical plate theory, is applicable to thin plates. Within this theory the effects of shear deformation and rotatory inertia are neglected resulting in the overestimation of vibration frequencies. This error becomes pronounced with the increase in the thickness of the plate. Thus, the classical plate theory produces accurate solutions until the thickness-to-length ratio reaches a value of about 1/20 [2]. Reissner [3] proposed a static theory taking into account the shear effect. In 1948 Uflyand [4], and several years later, in 1951, Mindlin [5] developed the thick plate theory or first-order shear deformation theory (FSDT) including effects of shear deformation and rotary inertia. Several decades later, Elishakoff [6] suggested a truncated version of this theory. A variational derivation based on slope inertia is also leading to a similar equation with an additional term in the governing differential equation [7] and in the boundary condition for a free edge. Since the last decades, the Uflyand-Mindlin plate model has been widely studied (see, for example, Irschik [8,9], Brunelle and Roberts [10], Brunelle [11], Sharma et al. [12]) and different methods were developed in order to determine the natural frequencies [13-16].

Liew et al. [13] presented the numerical p-version Ritz method and obtained the natural frequencies for different non-dimensional thickness. The Rayleigh-Ritz method is widely used in the literature. Introduced by Rayleigh [17] and further developed by Ritz [18], this method assumes that the displacement is a linear

F. Hache · I. Elishakoff

Department of Ocean and Mechanical Engineering, Florida Atlantic University, Boca Raton, FL 33481-0991, USA

E-mail: fhache2014@ fau.edu

I. Elishakoff

E-mail: elishako@fau.edu

F. Hache $\cdot$ N. Challamel $(\varangle)$

Université de Bretagne Sud, FRE CNRS 3744 IRDL, Centre de Recherche, Rue de Saint Maudé, BP 92116, 56321 Lorient, France

E-mail: noel.challamel@univ-ubs.fr 
combination of arbitrarily chosen admissible functions. Each amplitude coefficient associated with the functions must, according to the variational principle, minimize the energy functional. Since the last decades, many investigations are focused on the determination of appropriate functions [19-22]. The accuracy depends on the number of functions used in the series representing the displacement field, the expression of these functions; scientists have to find a compromise between accuracy and computational economy, as Liew et al. [13] mention.

Leissa [23] found the exact characteristic equations for thin rectangular plates having two opposite sides simply supported by using the Lévy approach. In this method, he distinguished the symmetric and antisymmetric modes. In addition to a better understanding of the vibrational mechanics, the problem is simplified and reduced to the determination of frequencies for which the determinant of a matrix $3 \times 3$ vanishes. Later, Gorman [24] generalized this work and developed the superposition method providing the natural frequencies for any boundary conditions. Later, Gorman and Ding [25] applied this method to obtain an exact expression of the solution for point supported Uflyand-Mindlin plates. Hashemi and Arsanjani [26] distinguished the symmetric and antisymmetric modes by using the Lévy approach, extending the work of Gorman to the free vibration of a thick plate.

The aim of this paper is to obtain the natural frequencies of a thick plate through the Lévy approach for the three alternative theories, namely (a) the original Uflyand-Mindlin plate theory, (b) the truncated UflyandMindlin plate theory, and (c) the Uflyand-Mindlin plate theory based on slope inertia, and to compare them with the results reported in the literature. Detailed numerical results are reported for all three versions for the first 10 natural frequencies for each of the six combinations of boundary conditions.

\section{Derivation of Uflyand-Mindlin plate models}

\subsection{Original and truncated Uflyand-Mindlin plate models}

It appears instructive to provide the variational derivation of the original Uflyand-Mindlin's equations presented in studies by Mindlin [5] and the equations of the Uflyand-Mindlin's theory based on slope inertia. Consider a thick rectangular plate of length $a$, width $b$, and uniform thickness $h$, as shown in Fig. 1. Hereinafter, plates with two opposite edges simply supported will be considered. For the purpose of description, a special notation will be adopted, the same as the one commonly used in the literature $[23,26,27]$ for the boundary conditions. The symbolism SCSF, for example, will identify a plate with the edges $x=0$ and $x=a$ simply supported, and the two others clamped and free [19] (see Fig. 3).

$\psi_{x}$ and $\psi_{y}$ are the bending rotations of a transverse normal about the $x$ and $y$ axis respectively, as shown in Fig. 2.

The potential energy is given in the following form:

$$
\begin{aligned}
V= & \iint_{\Omega} \frac{1}{2}\left(D\left\{\left(\frac{\partial \psi_{x}}{\partial x}+\frac{\partial \psi_{y}}{\partial y}\right)^{2}-2(1-v)\left[\frac{\partial \psi_{x}}{\partial x} \frac{\partial \psi_{y}}{\partial y}-\frac{1}{4}\left(\frac{\partial \psi_{x}}{\partial y}+\frac{\partial \psi_{y}}{\partial x}\right)^{2}\right]\right\}\right. \\
& \left.+\kappa^{2} G h\left[\left(\frac{\partial w}{\partial x}+\psi_{x}\right)^{2}+\left(\frac{\partial w}{\partial y}+\psi_{y}\right)^{2}\right]\right) d x d y
\end{aligned}
$$

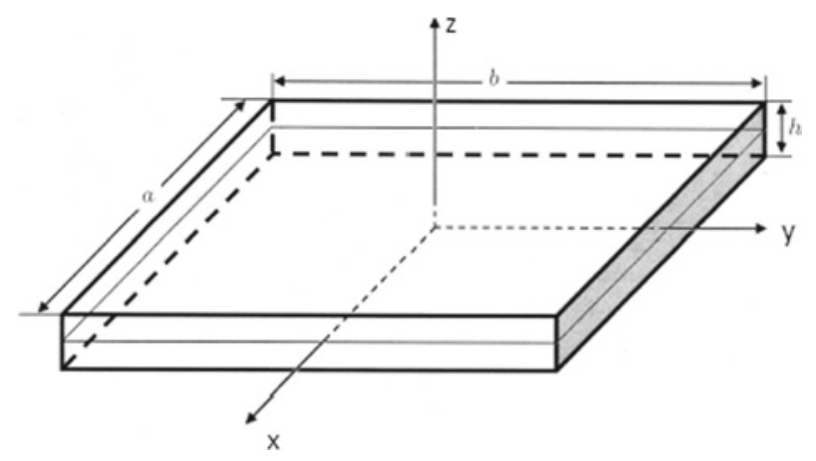

Fig. 1 Plate of dimensions $a \times b \times h$ 


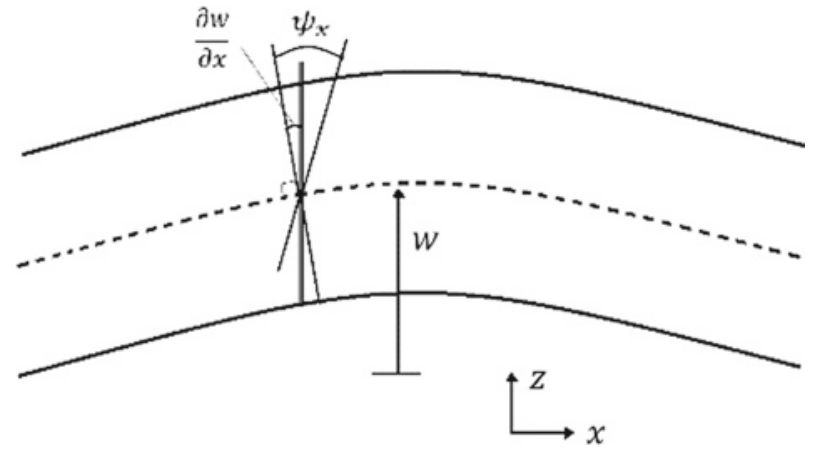

Fig. 2 Rotations of a transverse normal about the $y$ axis

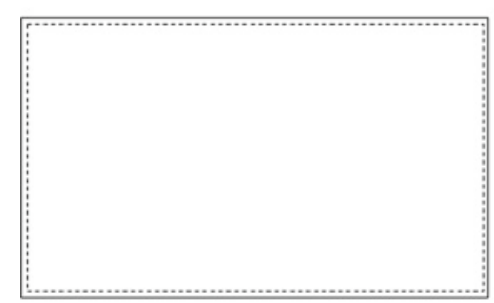

SSSS

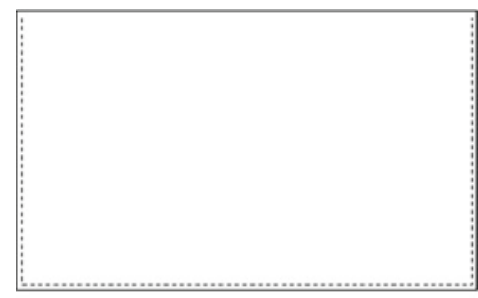

SFSS

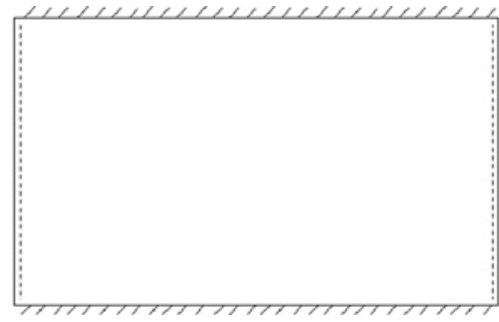

SCSC

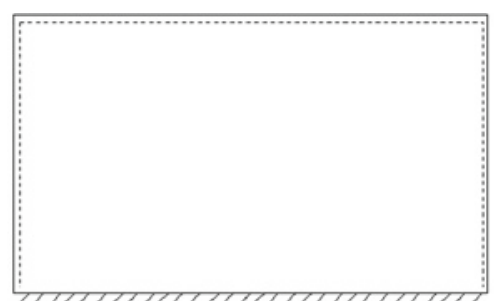

SSSC

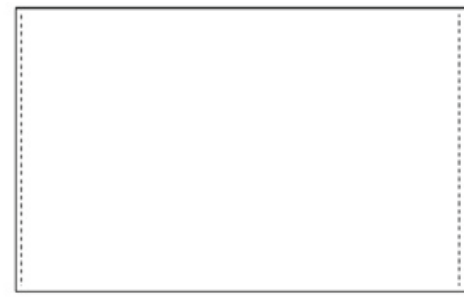

SFSF

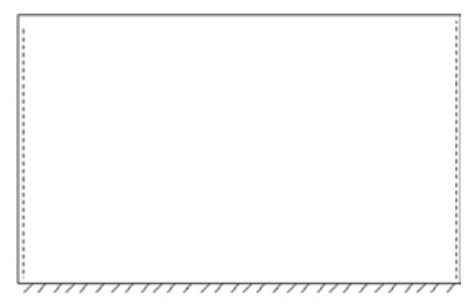

SFSC

Fig. 3 Set of considered boundary conditions: SSSS, SCSC, SFSF, SFSS, SSSC, SFSC

where $D=E h^{3} / 12\left(1-v^{2}\right)$ is the plate's flexural rigidity, $v$ the Poisson's ratio, $\kappa^{2}$ the shear coefficient, and $G$ the shear modulus of elasticity.

The expression of the kinetic energy is the following:

$$
T=\frac{1}{2} \iint_{\Omega} \rho h\left(\frac{\partial w}{\partial t}\right)^{2}+\frac{\rho h^{3}}{12}\left[\left(\frac{\partial \psi_{x}}{\partial t}\right)^{2}+\left(\frac{\partial \psi_{y}}{\partial t}\right)^{2}\right] d x d y,
$$

where $\Omega$ is the area of the mid-surface of the plate. Here it is worth noting that the term $V$ is responsible for the shear effect, whereas $T$ is associated with rotary inertia. Mindlin [5] and Liew et al. [13], when using the expression for kinetic energy, do not mention that de facto by using the expression $\left[\rho I\left(\partial \psi_{x} / \partial t\right)^{2}+\rho I\left(\partial \psi_{y} / \partial t\right)^{2}\right]$ they, in fact, correct the rotary inertia term which should be with the shear effect.

According to Hamilton's principle

$$
\delta \int_{t_{i}}^{t} \Pi d t=0
$$

where the Lagrangian $\Pi$ is given by:

$$
\Pi=T-V=\frac{1}{2} \iint_{\Omega}\left\{\frac{\rho h^{3}}{12}\left[\left(\frac{\partial \psi_{x}}{\partial t}\right)^{2}+\left(\frac{\partial \psi_{y}}{\partial t}\right)^{2}\right]+\rho h\left(\frac{\partial w}{\partial t}\right)^{2}\right\} d x d y
$$




$$
\begin{aligned}
& -\frac{1}{2} \iint_{\Omega}\left(D\left\{\left(\frac{\partial \psi_{x}}{\partial x}+\frac{\partial \psi_{y}}{\partial y}\right)^{2}-2(1-v)\left[\frac{\partial \psi_{x}}{\partial x} \frac{\partial \psi_{y}}{\partial y}-\frac{1}{4}\left(\frac{\partial \psi_{x}}{\partial y}+\frac{\partial \psi_{y}}{\partial x}\right)^{2}\right]\right\}\right. \\
& \left.+\kappa^{2} G h\left[\left(\frac{\partial w}{\partial x}+\psi_{x}\right)^{2}+\left(\frac{\partial w}{\partial y}+\psi_{y}\right)^{2}\right]\right) d x d y
\end{aligned}
$$

In view of Eqs. (3) and (4), one obtains:

$$
\begin{gathered}
\int_{t_{i}}^{t} \iint_{\Omega}\left\{-D\left(\frac{\partial \psi_{x}}{\partial x} \frac{\partial \delta \psi_{x}}{\partial x}+\frac{\partial \psi_{y}}{\partial y} \frac{\partial \delta \psi_{y}}{\partial y}+v \frac{\partial \psi_{x}}{\partial x} \frac{\partial \delta \psi_{y}}{\partial y}+v \frac{\partial \delta \psi_{x}}{\partial x} \frac{\partial \psi_{y}}{\partial y}\right)\right. \\
-\frac{D(1-v)}{2}\left(\frac{\partial \psi_{x}}{\partial y}+\frac{\partial \psi_{y}}{\partial x}\right)\left(\frac{\partial \delta \psi_{x}}{\partial y}+\frac{\partial \delta \psi_{y}}{\partial x}\right) \\
-\kappa^{2} G h\left[\left(\frac{\partial w}{\partial x}+\psi_{x}\right)\left(\frac{\partial \delta w}{\partial x}+\delta \psi_{x}\right)+\left(\frac{\partial w}{\partial y}+\psi_{y}\right)\left(\frac{\partial \delta w}{\partial y}+\delta \psi_{y}\right)\right] \\
\left.+\rho h \frac{\partial w}{\partial t} \frac{\partial \delta w}{\partial t}+\frac{\rho h^{3}}{12}\left(\frac{\partial \psi_{x}}{\partial t} \frac{\partial \delta \psi_{x}}{\partial t}+\frac{\partial \psi_{y}}{\partial t} \frac{\partial \delta \psi_{y}}{\partial t}\right)\right\} d x d y d t=0 .
\end{gathered}
$$

Integration by parts results in:

$$
\begin{aligned}
& \int_{t_{i}}^{t} \iint_{\Omega}\left\{D\left(\frac{\partial^{2} \psi_{x}}{\partial x^{2}} \delta \psi_{x}+\frac{\partial^{2} \psi_{y}}{\partial y^{2}} \delta \psi_{y}+v \frac{\partial^{2} \psi_{x}}{\partial x \partial y} \delta \psi_{y}+v \frac{\partial^{2} \psi_{y}}{\partial x \partial y} \delta \psi_{x}\right)\right. \\
& +\frac{D(1-v)}{2}\left(\frac{\partial^{2} \psi_{x}}{\partial y^{2}} \delta \psi_{x}+\frac{\partial^{2} \psi_{y}}{\partial x^{2}} \delta \psi_{y}+\frac{\partial^{2} \psi_{x}}{\partial x \partial y} \delta \psi_{y}+\frac{\partial^{2} \psi_{y}}{\partial x \partial y} \delta \psi_{x}\right) \\
& -\kappa^{2} G h\left[\left(\psi_{x} \delta \psi_{x}-\frac{\partial \psi_{x}}{\partial x} \delta w\right)+\left(\psi_{y} \delta \psi_{y}-\frac{\partial \psi_{y}}{\partial y} \delta w\right)+\left(\frac{\partial w}{\partial x} \delta \psi_{x}-\frac{\partial^{2} w}{\partial x^{2}} \delta w\right)\right. \\
& \left.\left.+\left(\frac{\partial w}{\partial y} \delta \psi_{y}-\frac{\partial^{2} w}{\partial y^{2}} \delta w\right)\right]-\rho h \frac{\partial^{2} w}{\partial t^{2}} \delta w-\frac{\rho h^{3}}{12}\left(\frac{\partial^{2} \psi_{x}}{\partial t^{2}} \delta \psi_{x}+\frac{\partial^{2} \psi_{y}}{\partial t^{2}} \delta \psi_{y}\right)\right\} d x d y d t \\
& -\int_{t_{i}}^{t} \oint\left\{D\left(\frac{\partial \psi_{x}}{\partial x} \delta \psi_{x} d y-\frac{\partial \psi_{y}}{\partial y} \delta \psi_{y} d x-v \frac{\partial \psi_{x}}{\partial x} \delta \psi_{y} d x+v \frac{\partial \psi_{y}}{\partial y} \delta \psi_{x} d y\right)\right. \\
& +\frac{D(1-v)}{2}\left(-\frac{\partial \psi_{x}}{\partial y} \delta \psi_{x} d y+\frac{\partial \psi_{x}}{\partial y} \delta \psi_{y} d y-\frac{\partial \psi_{y}}{\partial x} \delta \psi_{x} d x+\frac{\partial \psi_{y}}{\partial x} \delta \psi_{y} d y\right) \\
& \left.+\kappa^{2} G h\left(\psi_{x} \delta w d y+\frac{\partial w}{\partial x} \delta w d y-\psi_{y} \delta w d x-\frac{\partial w}{\partial y} \delta w d x\right)\right\} d t=0,
\end{aligned}
$$

where $\Gamma$ is the boundary path. Grouping the terms in the foregoing functional with respect to the variation terms yields

$$
\begin{aligned}
\int_{t_{i}}^{t} & \iint_{\Omega}\left\{\left[D\left(\frac{\partial^{2} \psi_{x}}{\partial x^{2}}+v \frac{\partial^{2} \psi_{y}}{\partial x \partial y}\right)+\frac{D(1-v)}{2}\left(\frac{\partial^{2} \psi_{x}}{\partial y^{2}}+v \frac{\partial^{2} \psi_{y}}{\partial x \partial y}\right)-\kappa^{2} G h\left(\psi_{x}+\frac{\partial w}{\partial x}\right)-\frac{\rho h^{3}}{12} \frac{\partial^{2} \psi_{x}}{\partial t^{2}}\right] \delta \psi_{x}\right. \\
+ & {\left[D\left(\frac{\partial^{2} \psi_{y}}{\partial y^{2}}+v \frac{\partial^{2} \psi_{x}}{\partial x \partial y}\right)+\frac{D(1-v)}{2}\left(\frac{\partial^{2} \psi_{y}}{\partial x^{2}}+v \frac{\partial^{2} \psi_{x}}{\partial x \partial y}\right)-\kappa^{2} G h\left(\psi_{y}+\frac{\partial w}{\partial y}\right)-\frac{\rho h^{3}}{12} \frac{\partial^{2} \psi_{y}}{\partial t^{2}}\right] \delta \psi_{y} } \\
+ & {\left.\left[\kappa^{2} G h\left(\frac{\partial \psi_{x}}{\partial x}+\frac{\partial^{2} w}{\partial x^{2}}+\frac{\partial \psi_{y}}{\partial y}+\frac{\partial^{2} w}{\partial y^{2}}\right)-\rho h \frac{\partial^{2} w}{\partial t^{2}}\right] \delta w\right\} d x d y d t } \\
- & \int_{t_{i}}^{t} \oint_{\Gamma}\left[\left\{D\left(\frac{\partial \psi_{x}}{\partial x} d y+v \frac{\partial \psi_{y}}{\partial y} d y\right)-\frac{D(1-v)}{2}\left(\frac{\partial \psi_{x}}{\partial y} d x+\frac{\partial \psi_{y}}{\partial x} d x\right)\right\} \delta \psi_{x}\right.
\end{aligned}
$$




$$
\begin{aligned}
& +\left\{-D\left(\frac{\partial \psi_{y}}{\partial y} d x+v \frac{\partial \psi_{x}}{\partial x} d x\right)+\frac{D(1-v)}{2}\left(\frac{\partial \psi_{x}}{\partial y} d y+\frac{\partial \psi_{y}}{\partial x} d y\right)\right\} \delta \psi_{y} \\
& \left.+\kappa^{2} G h\left(\psi_{x} d y+\frac{\partial w}{\partial x} d y-\psi_{y} d x-\frac{\partial w}{\partial y} d x\right) \delta w\right] d t=0 .
\end{aligned}
$$

Equating the coefficients of the variation terms to zero for the functional over the plate area, the equations of motions are obtained $[8,16]$ as

$$
\begin{gathered}
\frac{D}{2}\left[(1-v) \nabla^{2} \psi_{x}+(1+v)\left(\frac{\partial^{2} \psi_{x}}{\partial x^{2}}+\frac{\partial \psi_{y}}{\partial x \partial y}\right)\right]-\kappa^{2} G h\left(\psi_{x}+\frac{\partial w}{\partial x}\right)=\frac{\rho h^{3}}{12} \frac{\partial^{2} \psi_{x}}{\partial t^{2}}, \\
\frac{D}{2}\left[(1-v) \nabla^{2} \psi_{y}+(1+v)\left(\frac{\partial \psi_{x}}{\partial x \partial y}+\frac{\partial^{2} \psi_{y}}{\partial y^{2}}\right)\right]-\kappa^{2} G h\left(\psi_{y}+\frac{\partial w}{\partial y}\right)=\frac{\rho h^{3}}{12} \frac{\partial^{2} \psi_{y}}{\partial t^{2}}, \\
\kappa^{2} G h\left(\nabla^{2} w+\frac{\partial \psi_{x}}{\partial x}+\frac{\partial \psi_{y}}{\partial y}\right)=\rho h \frac{\partial^{2} w}{\partial t^{2}} .
\end{gathered}
$$

Some algebraic manipulations lead to the governing differential equation in terms of the plate's displacement:

$$
D \nabla^{4} w+\rho h \frac{\partial^{2} w}{\partial t^{2}}-\rho \frac{h^{3}}{12}\left(1+\frac{12}{h^{3}} \frac{D}{\kappa^{2} G}\right) \frac{\partial^{2}}{\partial t^{2}} \nabla^{2} w+\frac{\rho^{2} h^{3}}{12 \kappa^{2} G} \frac{\partial^{4} w}{\partial t^{4}}=0 .
$$

In his paper, Elishakoff, Hache and Challamel [7], following Elishakoff [6] for the Timoshenko beams, suggested that $\partial^{2} \psi_{x} / \partial t^{2}$ and $\partial^{2} \psi_{y} / \partial t^{2}$ in Eqs. (8) and (9) ought to be, respectively, replaced by $\partial^{3} w / \partial x \partial t^{2}$ and $\partial^{3} w / \partial y \partial t^{2}$ for Mindlin plates.

After some algebraic manipulations, a truncated simpler governing differential equation is obtained:

$$
D \nabla^{4} w+\rho h \frac{\partial^{2} w}{\partial t^{2}}-\rho \frac{h^{3}}{12}\left(1+\frac{12}{h^{3}} \frac{D}{\kappa^{2} G}\right) \frac{\partial^{2}}{\partial t^{2}} \nabla^{2} w=0
$$

instead of Eq. (11). This truncated Mindlin plate model can be supported by asymptotic arguments from 3d-elasticity, as it is proven for the truncated Bresse-Timoshenko model [28].

In the case where $12 D / h^{3} \kappa^{2} G \gg 1$, one obtains the reduced truncated Mindlin plate equation:

$$
D \nabla^{4} w+\rho h \frac{\partial^{2} w}{\partial t^{2}}-\rho \frac{D}{\kappa^{2} G} \frac{\partial^{2}}{\partial t^{2}} \nabla^{2} w=0 .
$$

Note that this equation is also obtained by Mindlin [28] without any comment on the benefit or drawback in comparison with original Uflyand [4] and Mindlin [5] derivations of Eq. (11). For boundary conditions, the line integral of Eq. (6) is set to zero and rewritten as:

$$
\begin{aligned}
& \int_{t_{i}}^{t} \oint_{\Gamma}\left[D\left(\frac{\partial \psi_{x}}{\partial x}+v \frac{\partial \psi_{y}}{\partial y}\right) \delta \psi_{x} d y-D\left(\frac{\partial \psi_{y}}{\partial y}+v \frac{\partial \psi_{x}}{\partial x}\right) \delta \psi_{y} d x+\frac{D(1-v)}{2}\left(\frac{\partial \psi_{x}}{\partial y}+\frac{\partial \psi_{y}}{\partial x}\right) \delta \psi_{y} d y\right. \\
& \left.-\frac{D(1-v)}{2}\left(\frac{\partial \psi_{x}}{\partial y}+\frac{\partial \psi_{y}}{\partial x}\right) \delta \psi_{x} d x+\kappa^{2} G h\left(\psi_{x}+\frac{\partial w}{\partial x}\right) \delta w d y-\kappa^{2} G h\left(\psi_{y}+\frac{\partial w}{\partial y}\right) \delta w d x\right] d t=0 .
\end{aligned}
$$

At the boundaries of the plate, for edges parallel to the $x$ axis,

$$
\begin{gathered}
D\left(\frac{\partial \psi_{x}}{\partial x}+v \frac{\partial \psi_{y}}{\partial y}\right)=0 \text { or } \psi_{x} \\
\frac{\partial \psi_{x}}{\partial y}+\frac{\partial \psi_{y}}{\partial x}=0 \text { or } \psi_{y} \\
\kappa^{2} G h\left(\psi_{y}+\frac{\partial w}{\partial y}\right)=0 \text { or } w
\end{gathered}
$$

are specified. For edges parallel to the $y$ axis,

$$
\begin{gathered}
D\left(\frac{\partial \psi_{y}}{\partial y}+v \frac{\partial \psi_{x}}{\partial x}\right)=0 \text { or } \psi_{y} \\
\frac{\partial \psi_{x}}{\partial y}+\frac{\partial \psi_{y}}{\partial x}=0 \text { or } \psi_{x} \\
\kappa^{2} G h\left(\psi_{x}+\frac{\partial w}{\partial x}\right)=0 \text { or } w
\end{gathered}
$$

are specified. It is assumed in this case that the boundary conditions of the truncated Mindlin plate model and the original Mindlin plate model are the same. 


\subsection{Uflyand-Mindlin plate model based on slope inertia}

In his paper, Mindlin [5] uses the exact expression of the kinetic energy in three dimensions given by the general linear theory of elasticity. By using Eq. (2) which contains also a correction in order to take into account the shear effect, Mindlin "overcorrected," as it were, the kinetic energy. It is suggested [7] to replace the expression of the kinetic energy given in Eq. (2) by:

$$
T=\frac{1}{2} \iint \frac{\rho h^{3}}{12}\left[\left(\frac{\partial^{2} w}{\partial t \partial x}\right)^{2}+\left(\frac{\partial^{2} w}{\partial t \partial y}\right)^{2}\right]+\rho h\left(\frac{\partial w}{\partial t}\right)^{2} d x d y .
$$

Substituting Eqs. (1) and (17) into Eq. (3) yields

$$
\begin{gathered}
\int_{t_{i}}^{t} \iint_{\Omega}\left\{-D\left(\frac{\partial \psi_{x}}{\partial x} \frac{\partial \delta \psi_{x}}{\partial x}+\frac{\partial \psi_{y}}{\partial y} \frac{\partial \delta \psi_{y}}{\partial y}+v \frac{\partial \psi_{x}}{\partial x} \frac{\partial \delta \psi_{y}}{\partial y}+v \frac{\partial \delta \psi_{x}}{\partial x} \frac{\partial \psi_{y}}{\partial y}\right)\right. \\
-\frac{D(1-v)}{2}\left(\frac{\partial \psi_{x}}{\partial y}+\frac{\partial \psi_{y}}{\partial x}\right)\left(\frac{\partial \delta \psi_{x}}{\partial y}+\frac{\partial \delta \psi_{y}}{\partial x}\right) \\
-\kappa^{2} G h\left[\left(\frac{\partial w}{\partial x}+\psi_{x}\right)\left(\frac{\partial \delta w}{\partial x}+\delta \psi_{x}\right)+\left(\frac{\partial w}{\partial y}+\psi_{y}\right)\left(\frac{\partial \delta w}{\partial y}+\delta \psi_{y}\right)\right] \\
\left.+\rho h \frac{\partial w}{\partial t} \frac{\partial \delta w}{\partial t}+\frac{\rho h^{3}}{12}\left(\frac{\partial^{2} w}{\partial t \partial x} \frac{\partial^{2} \delta w}{\partial t \partial x}+\frac{\partial^{2} w}{\partial t \partial y} \frac{\partial^{2} \delta w}{\partial t \partial t y}\right)\right\} d x d y d t=0 .
\end{gathered}
$$

Integrating by parts yields

$$
\begin{aligned}
& \int_{t_{i}}^{t} \iint_{\Omega}\left\{\left[D\left(\frac{\partial^{2} \psi_{x}}{\partial x^{2}}+v \frac{\partial^{2} \psi_{y}}{\partial x \partial y}\right)+\frac{D(1-v)}{2}\left(\frac{\partial^{2} \psi_{x}}{\partial y^{2}}+v \frac{\partial^{2} \psi_{y}}{\partial x \partial y}\right)-\kappa^{2} G h\left(\psi_{x}+\frac{\partial w}{\partial x}\right)\right] \delta \psi_{x}\right. \\
& +\left[D\left(\frac{\partial^{2} \psi_{y}}{\partial y^{2}}+v \frac{\partial^{2} \psi_{x}}{\partial x \partial y}\right)+\frac{D(1-v)}{2}\left(\frac{\partial^{2} \psi_{y}}{\partial x^{2}}+v \frac{\partial^{2} \psi_{x}}{\partial x \partial y}\right)-\kappa^{2} G h\left(\psi_{y}+\frac{\partial w}{\partial y}\right)\right] \delta \psi_{y} \\
& \left.+\left[\kappa^{2} G h\left(\frac{\partial \psi_{x}}{\partial x}+\frac{\partial^{2} w}{\partial x^{2}}+\frac{\partial \psi_{y}}{\partial y}+\frac{\partial^{2} w}{\partial y^{2}}\right)-\rho h \frac{\partial^{2} w}{\partial t^{2}}+\frac{\rho h^{3}}{12} \frac{\partial^{2}}{\partial t^{2}} \nabla^{2} w\right] \delta w\right\} d x d y d t \\
& +\int_{t_{i}}^{t} \oint_{\Gamma}\left[\left\{D\left(\frac{\partial \psi_{x}}{\partial x} d y+v \frac{\partial \psi_{y}}{\partial y} d y\right)-\frac{D(1-v)}{2}\left(\frac{\partial \psi_{x}}{\partial y} d x+\frac{\partial \psi_{y}}{\partial x} d x\right)\right\} \delta \psi_{x}\right. \\
& +\left\{-D\left(\frac{\partial \psi_{y}}{\partial y} d x+v \frac{\partial \psi_{x}}{\partial x} d x\right)+\frac{D(1-v)}{2}\left(\frac{\partial \psi_{x}}{\partial y} d y+\frac{\partial \psi_{y}}{\partial x} d y\right)\right\} \delta \psi_{x} \\
& +\kappa^{2} G h\left(\psi_{x} d y+\frac{\partial w}{\partial x} d y-\psi_{y} d x-\frac{\partial w}{\partial y} d x\right) \delta w \\
& \left.+\frac{\rho h^{3}}{12}\left(\frac{\partial^{2} w}{\partial t^{2} \partial x} d y+\frac{\partial^{2} w}{\partial t^{2} \partial y} d x\right) \delta w\right] d t=0 .
\end{aligned}
$$

Equating the coefficients of the variation terms to zero for the functional over the plate area, the equations of motion are obtained as follows:

$$
\begin{gathered}
\frac{D}{2}\left[(1-v) \nabla^{2} \psi_{x}+(1+v)\left(\frac{\partial^{2} \psi_{x}}{\partial x^{2}}+\frac{\partial \psi_{y}}{\partial x \partial y}\right)\right]-\kappa^{2} G h\left(\psi_{x}+\frac{\partial w}{\partial x}\right)=0, \\
\frac{D}{2}\left[(1-v) \nabla^{2} \psi_{y}+(1+v)\left(\frac{\partial \psi_{x}}{\partial x \partial y}+\frac{\partial^{2} \psi_{y}}{\partial y^{2}}\right)\right]-\kappa^{2} G h\left(\psi_{y}+\frac{\partial w}{\partial y}\right)=0, \\
\kappa^{2} G h\left(\nabla^{2} w+\frac{\partial \psi_{x}}{\partial x}+\frac{\partial \psi_{y}}{\partial y}\right)=\rho h\left(1-\frac{h^{2}}{12} \nabla^{2}\right) \frac{\partial^{2} w}{\partial t^{2}} .
\end{gathered}
$$

From Eqs. (20) to (22), the governing equation is obtained:

$$
D \nabla^{4} w+\rho h \frac{\partial^{2} w}{\partial t^{2}}-\frac{\rho h^{3}}{12}\left(1+\frac{12}{h^{3}} \frac{D}{\kappa^{2} G}\right) \frac{\partial^{2}}{\partial t^{2}} \nabla^{2} w+\frac{\rho h^{2} D}{12 \kappa^{2} G} \frac{\partial^{2}}{\partial t^{2}} \nabla^{4} w=0 .
$$


The difference with original Uflyand-Mindlin equations is twofold: (a) an additional, the last, term occurs, and (b) the fourth-order time derivative, that is characteristic of the original Uflyand-Mindlin plate theory, does not appear.

For boundary conditions, the line integral of Eq. (19) is set to zero and rewritten as:

$$
\begin{aligned}
& \int_{t_{i}}^{t} \oint_{\Gamma}\left[\left\{D\left(\frac{\partial \psi_{x}}{\partial x}+v \frac{\partial \psi_{y}}{\partial y}\right) d y-\frac{D(1-\mu)}{2}\left(\frac{\partial \psi_{x}}{\partial y}+\frac{\partial \psi_{y}}{\partial x}\right) d x\right\} \delta \psi_{x}\right. \\
& +\left\{-D\left(\frac{\partial \psi_{y}}{\partial y}+v \frac{\partial \psi_{x}}{\partial x}\right) d x+\frac{D(1-v)}{2}\left(\frac{\partial \psi_{x}}{\partial y}+\frac{\partial \psi_{y}}{\partial x}\right) d y\right\} \delta \psi_{y} \\
& \left.\quad+\left[\left(\frac{\rho h^{3}}{12} \frac{\partial^{2} w}{\partial t^{2} \partial x}+\kappa^{2} G h \psi_{x}+\kappa^{2} G h \frac{\partial w}{\partial x}\right) d y-\left(\frac{\rho h^{3}}{12} \frac{\partial^{2} w}{\partial t^{2} \partial y}+\kappa^{2} G h \psi_{y}-\kappa^{2} G h \frac{\partial w}{\partial y}\right) d x\right] \delta w\right] d t=0 .
\end{aligned}
$$

Equation (24) implies the boundaries of the plate.

For edges parallel to the $x$ axis,

$$
\begin{gathered}
D\left(\frac{\partial \psi_{x}}{\partial x}+v \frac{\partial \psi_{y}}{\partial y}\right)=0 \text { or } \psi_{x} \\
\frac{\partial \psi_{x}}{\partial y}+\frac{\partial \psi_{y}}{\partial x}=0 \text { or } \psi_{y} \\
\kappa^{2} G h\left(\psi_{y}+\frac{\partial w}{\partial y}\right)-\frac{\rho h^{3}}{12} \frac{\partial^{2} w}{\partial t^{2} \partial y}=0 \text { or } w
\end{gathered}
$$

are specified. For edges parallel to the $y$ axis,

$$
\begin{gathered}
D\left(\frac{\partial \psi_{y}}{\partial y}+v \frac{\partial \psi_{x}}{\partial x}\right)=0 \text { or } \psi_{y} \\
\frac{\partial \psi_{x}}{\partial y}+\frac{\partial \psi_{y}}{\partial x}=0 \text { or } \psi_{x} \\
\kappa^{2} G h\left(\psi_{x}+\frac{\partial w}{\partial x}\right)+\frac{\rho h^{3}}{12} \frac{\partial^{2} w}{\partial t^{2} \partial x}=0 \text { or } w
\end{gathered}
$$

are specified. When the edge is simply supported or clamped, the boundary conditions at the edge match the ones given by the original and the truncated Uflyand-Mindlin plate theory.

\section{Transformation of governing differential equations}

Introducing control parameters $\gamma_{1}, \gamma_{2}$, and $\gamma_{3}$, with $\left(\gamma_{1}, \gamma_{2}, \gamma_{3}\right)$ equal to $(1,0,0),(0,0,1)$ and $(0,1,0)$ for the original Uflyand-Mindlin plate theory, the truncated Uflyand-Mindlin theory and the Uflyand-Mindlin model based on slope inertia, also denoted UM, truncated UM, and slope inertia UM, respectively, the equations of motion are given as follows for all Uflyand-Mindlin plate models, setting $C=D(1-v) / 2$ :

$$
\begin{gathered}
D \frac{\partial}{\partial x}\left(\frac{\partial \psi_{x}}{\partial x}+\frac{\partial \psi_{y}}{\partial y}\right)+C \frac{\partial}{\partial y}\left(\frac{\partial \psi_{x}}{\partial y}-\frac{\partial \psi_{y}}{\partial x}\right)-\kappa^{2} G h\left(\psi_{x}+w_{, x}\right)=\gamma_{1} \frac{\rho h^{3}}{12} \frac{\partial^{2}}{\partial t^{2}} \psi_{x}+\gamma_{3} \frac{\rho h^{3}}{12} \frac{\partial^{3} w}{\partial x \partial t^{2}} \\
D \frac{\partial}{\partial y}\left(\frac{\partial \psi_{x}}{\partial x}+\frac{\partial \psi_{y}}{\partial y}\right)-C \frac{\partial}{\partial x}\left(\frac{\partial \psi_{x}}{\partial y}-\frac{\partial \psi_{y}}{\partial x}\right)-\kappa^{2} G h\left(\psi_{y}+w_{, y}\right)=\gamma_{1} \frac{\rho h^{3}}{12} \frac{\partial^{2} \psi_{y}}{\partial t^{2}}+\gamma_{3} \frac{\rho h^{3}}{12} \frac{\partial^{3} w}{\partial y \partial t^{2}} \\
\kappa^{2} G h\left(\frac{\partial \psi_{x}}{\partial x}+\frac{\partial \psi_{y}}{\partial y}+\frac{\partial^{2} w}{\partial x^{2}}+\frac{\partial^{2} w}{\partial y^{2}}\right)=\left(\rho h-\gamma_{2} \frac{\rho h^{3}}{12} \nabla^{2}\right) \frac{\partial^{2} w}{\partial t^{2}} .
\end{gathered}
$$

In order to solve these three coupled partial differential equations, it is more convenient to deal with uncoupled equations $[26,27,32]$. The governing equations can be rewritten as:

$$
\begin{aligned}
& D \varsigma_{, x}+C \varphi_{, y}-\kappa^{2} G h\left(\psi_{x}+w_{, x}\right)=\gamma_{1} \frac{\rho h^{3}}{12} \ddot{\psi}_{x}+\gamma_{3} \frac{\rho h^{3}}{12} \frac{\partial^{3} w}{\partial x \partial t^{2}}, \\
& D \varsigma_{, y}-C \varphi_{, x}-\kappa^{2} G h\left(\psi_{y}+w_{, y}\right)=\gamma_{1} \frac{\rho h^{3}}{12} \ddot{\psi}_{y}+\gamma_{3} \frac{\rho h^{3}}{12} \frac{\partial^{3} w}{\partial y \partial t^{2}}, \\
& \kappa^{2} G h\left(\psi_{x, x}+\psi_{y, y}+w_{, x x}+w_{, y y}\right)=\left(\rho h-\gamma_{2} \frac{\rho h^{3}}{12} \nabla\right) \ddot{w},
\end{aligned}
$$


where the function $\varphi$ is introduced as [32]:

$$
\varphi=\psi_{x, y}-\psi_{y, x}
$$

Differentiating Eqs. (30) and (31) with respect to $y$ and $x$, respectively, and subtracting Eq. (31) from (30), one obtains:

$$
C \nabla^{2}\left(\psi_{x, y}-\psi_{y, x}\right)-\kappa^{2} G h\left(\psi_{x, y}-\psi_{y, x}\right)=\gamma_{1} \frac{\rho h^{3}}{12} \frac{\partial^{2}}{\partial t^{2}}\left(\psi_{x, y}-\psi_{y, x}\right)
$$

This yields

$$
C \nabla^{2} \varphi-\kappa^{2} G h \varphi=\gamma_{1} \frac{\rho h^{3}}{12} \frac{\partial^{2}}{\partial t^{2}} \varphi
$$

Moreover, Eqs. (11), (12), and (23) are written in general form as follows [7]:

$$
D\left(1+\gamma_{2} \frac{\rho h^{2}}{12 \kappa^{2} G} \frac{\partial^{2}}{\partial t^{2}}\right) \nabla^{4} w+\rho h \frac{\partial^{2} w}{\partial t^{2}}-\rho \frac{h^{3}}{12}\left(1+\frac{12}{h^{3}} \frac{D}{\kappa^{2} G}\right) \frac{\partial^{2}}{\partial t^{2}} \nabla^{2} w+\gamma_{1} \frac{\rho^{2} h^{3}}{12 \kappa^{2} G} \frac{\partial^{4} w}{\partial t^{4}}=0
$$

Thus, the system of three coupled equations is reduced to a system of two uncoupled equations. The solution of such a system is known.

Moreover, the rotation angles $\psi_{x}$ and $\psi_{y}$ can be found from Eqs. (30) to (31) by the following relation:

$$
\begin{aligned}
& \left(\kappa^{2} G h-\gamma_{1} \frac{\rho h^{3}}{12} \omega^{2}\right) \psi_{x}=\frac{\partial}{\partial x}\left[-\rho \omega^{2} \frac{D\left(1-\gamma_{2} \frac{h^{2}}{12} \nabla^{2}\right)}{\kappa^{2} G} w-D \nabla^{2} w-\kappa^{2} G h w\right]+C \frac{\partial \varphi}{\partial y}+\gamma_{3} \frac{\rho h^{3}}{12} \omega^{2} \frac{\partial w}{\partial x}, \\
& \left(\kappa^{2} G h-\gamma_{1} \frac{\rho h^{3}}{12} \omega^{2}\right) \psi_{y}=\frac{\partial}{\partial y}\left[-\rho \omega^{2} \frac{D\left(1-\gamma_{2} \frac{h^{2}}{12} \nabla^{2}\right)}{\kappa^{2} G} w-D \nabla^{2} w-\kappa^{2} G h w\right]-C \frac{\partial \varphi}{\partial x}+\gamma_{3} \frac{\rho h^{3}}{12} \omega^{2} \frac{\partial w}{\partial y} .
\end{aligned}
$$

It is assumed that

$$
\left(w ; \psi_{x} ; \psi_{y} ; \varphi\right)(x, y, t)=\left(\bar{w} ; \bar{\psi}_{x} ; \bar{\psi}_{y} ; \bar{\varphi}\right)(x, y) e^{i \omega t} .
$$

Substituting Eq. (39) into Eqs. (35) and (36) leads to:

$$
\begin{gathered}
C \nabla^{2} \bar{\varphi}-\left(\kappa^{2} G h-\gamma_{1} \frac{\rho h^{3}}{12} \omega^{2}\right) \bar{\varphi}=0 \\
D\left(1-\frac{\rho h^{2}}{12 \kappa^{2} G} \omega^{2} \gamma_{2}\right) \nabla^{4} \bar{w}+\rho \frac{h^{3}}{12}\left(1+\frac{12}{h^{3}} \frac{D}{\kappa^{2} G}\right) \omega^{2} \nabla^{2} \bar{w}+\left(\gamma_{1} \frac{\rho^{2} h^{3}}{12 \kappa^{2} G} \omega^{2}-\rho h\right) \omega^{2} \bar{w}=0 .
\end{gathered}
$$

\section{Rectangular plates with four edges simply supported}

Consider a plate with all the edges simply supported (Fig. 3a). The following non-dimensional numbers are defined:

$$
\lambda=\omega b^{2} \sqrt{\frac{\rho h}{D}} ; \beta=\frac{E}{G\left(1-v^{2}\right)} ; y=\eta b=\frac{\eta}{\chi} a ; \bar{h}=\frac{h}{a} ; \xi=\frac{x}{a} ; \chi=\frac{a}{b} .
$$

In view of the boundary conditions, the solution is given by Navier [29] as

$$
\bar{w}(x, y)=a \sin (n \pi \eta) \sin (m \pi \xi),
$$

where $m$ and $n$ are the numbers of half-waves in the $x$ and $y$ direction, respectively.

Substituting Eqs. (42) and (43) into Eq. (41), 


$$
\begin{aligned}
& {\left[(\chi n)^{2}+m^{2}\right]^{2} \pi^{4}-\chi^{4} \lambda^{2}\left[1+\frac{\bar{h}^{2} \pi^{2}}{12}\left(1+\frac{\beta}{\kappa^{2}}\right)\left[(\chi n)^{2}+m^{2}\right]+\left[(\chi n)^{2}+m^{2}\right]^{2} \frac{\beta \bar{h}^{4} \pi^{4}}{144 \kappa^{2}} \gamma_{2}\right]} \\
& \quad+\frac{\beta \chi^{8} \bar{h}^{4}}{144 \kappa^{2}} \gamma_{1} \lambda^{4}=0 .
\end{aligned}
$$

For the original Uflyand-Mindlin model, $\gamma_{1}=1$ and $\gamma_{2}=0$. The solution of Eq. (45) is expressed as:

$$
\begin{aligned}
\lambda= & \frac{12}{\chi^{2} \bar{h}^{2}} \sqrt{\frac{\kappa^{2}}{2 \beta}}\left(1+\frac{\bar{h}^{2} \pi^{2}}{12}\left(1+\frac{\beta}{\kappa^{2}}\right)\left[(\chi n)^{2}+m^{2}\right]\right. \\
& \left. \pm \sqrt{\left[1+\frac{\bar{h}^{2} \pi^{2}}{12}\left(1+\frac{\beta}{\kappa^{2}}\right)\left[(\chi n)^{2}+m^{2}\right]\right]^{2}-\frac{\beta \pi^{4} \bar{h}^{4}}{36 \kappa^{2}}\left[(\chi n)^{2}+m^{2}\right]^{2}}\right)^{\frac{1}{2}} .
\end{aligned}
$$

It is seen that the natural frequency is given by two different expressions. The second expression is associated with a second branch of frequencies.

This result matches with the one of Irschik [8], Wang [34], and Wang and Wang [36]. For the truncated Uflyand-Mindlin model and the slope inertia-based Uflyand-Mindlin model, the natural frequencies are given by:

$$
\left[(\chi n)^{2}+m^{2}\right]^{2} \pi^{4}-\chi^{4} \lambda^{2}\left[1+\frac{\bar{h}^{2} \pi^{2}}{12}\left(1+\frac{\beta}{\kappa^{2}}\right)\left[(\chi n)^{2}+m^{2}\right]+\left[(\chi n)^{2}+m^{2}\right]^{2} \frac{\beta \bar{h}^{4} \pi^{4}}{144 \kappa^{2}} \gamma_{2}\right]=0 .
$$

From Eq. (47), one obtains

$$
\lambda=\frac{\left[(\chi n)^{2}+m^{2}\right] \pi^{2}}{\chi^{2} \sqrt{1+\frac{\bar{h}^{2} \pi^{2}}{12}\left(1+\frac{\beta}{\kappa^{2}}\right)\left[(\chi n)^{2}+m^{2}\right]+\left[(\chi n)^{2}+m^{2}\right]^{2} \frac{\beta \bar{h}^{4} \pi^{4}}{144 \kappa^{2}} \gamma_{2}}} .
$$

Since $\gamma_{2}$ equals zero for the truncated model and equals unity for the slope inertia-based model, the Uflyand-Mindlin model based on slope inertia provides smaller values for natural frequencies than does the truncated Uflyand-Mindlin model. The study of the natural frequencies for the plates having simply supported two opposite edges will show that this result is valid also to other sets of boundary conditions. Moreover, it is worth noting that in contrast to the original Uflyand-Mindlin plate theory, these two models provide one unique branch of frequencies. Therefore, the results provided by these two theories can be compared with the frequencies predicted by the first branch of the original Uflyand-Mindlin theory.

\section{Solutions for two opposite simply supported edges: Lévy's approach}

Consider a plate with simple supports along the edges $\xi=0$ and $\xi=1$. Following the approach developed by Lévy (see, for instance, Chen and Liu [30], Szilard [31], and Jomehzadeh [32]):

$$
\bar{w}(\xi, \eta)=\sum_{m=1}^{\infty} a W_{m}(\eta) \sin (m \pi \xi) ; \bar{\psi}_{x}(\xi, \eta)=\sum_{m=1}^{\infty} \phi_{x}(\eta) \cos (m \pi \xi) ; \bar{\psi}_{y}(\xi, \eta)=\sum_{m=1}^{\infty} \phi_{y}(\eta) \sin (m \pi \xi) .
$$

Consequently, using Eq. (33),

$$
\bar{\varphi}(\xi, \eta)=\frac{1}{a} \sum_{m=1}^{\infty} \varphi_{m}(\eta) \cos (m \pi \xi) .
$$

Substituting Eqs. (48) and (49) into Eqs. (40) and (41) leads to the non-dimensional equations of motion:

$$
\Lambda_{1} \frac{d^{4} W_{m}}{d \eta^{4}}+\Lambda_{2} \frac{d^{2} W_{m}}{d \eta^{2}}+\Lambda_{3} W_{m}=0
$$




$$
\Lambda_{4} \frac{d^{2} \varphi_{m}}{d \eta^{2}}+\Lambda_{5} \varphi_{m}=0
$$

where the material constants $\Lambda_{i}(i=1, \ldots, 5)$ are given by

$$
\begin{gathered}
\Lambda_{1}=\chi^{4}\left(1-\gamma_{2} \frac{\beta \chi^{4} \bar{h}^{4}}{144 \kappa^{2}} \lambda^{2}\right), \\
\Lambda_{2}=\left[\chi^{6} \lambda^{2} \frac{\bar{h}^{2}}{12}\left(1+\frac{\beta}{\kappa^{2}}\right)-2(\alpha m \pi)^{2}\left(1-\frac{\beta \chi^{4} \bar{h}^{4}}{144 \kappa^{2}} \lambda^{2} \gamma_{2}\right)\right], \\
\Lambda_{3}=\left[(m \pi)^{4}-\chi^{4} \lambda^{2}\left[(m \pi)^{4} \frac{\beta \bar{h}^{4}}{144 \kappa^{2}} \gamma_{2}+\frac{(m \pi \bar{h})^{2}}{12}\left(1+\frac{\beta}{\kappa^{2}}\right)+1\right]+\frac{\beta \chi^{8} \bar{h}^{4}}{144 \kappa^{2}} \gamma_{1} \lambda^{4}\right], \\
\Lambda_{4}=\beta \chi^{2} \frac{\bar{h}^{2}}{24}(1-v), \\
\Lambda_{5}=-\left[(m \pi)^{2} \beta \frac{\bar{h}^{2}}{24}(1-v)+\kappa^{2}-\gamma_{1} \frac{\beta \chi^{4} \bar{h}^{4}}{144} \lambda^{2}\right],
\end{gathered}
$$

and Eqs. (37) and (38) become:

$$
\begin{aligned}
\left(\kappa^{2}\right. & \left.-\gamma_{1} \lambda^{2} \beta \frac{\chi^{4} \bar{h}^{4}}{144}\right) \psi_{x} \\
= & -m \pi\left[\left[\frac{\beta^{2} \chi^{4} \bar{h}^{4}}{144} \lambda^{2} \frac{1}{\kappa^{2}}\left(1+(m \pi)^{2} \gamma_{2} \frac{\bar{h}^{2}}{12}\right)-(m \pi)^{2} \frac{\beta \bar{h}^{2}}{12}+\kappa^{2}-\gamma_{3} \lambda^{2} \beta \frac{\chi^{4} \bar{h}^{4}}{144}\right] W\right. \\
& \left.+\left[\chi^{2} \frac{\beta \bar{h}^{2}}{12}-\frac{\beta^{2} \chi^{6} \bar{h}^{6}}{1728} \lambda^{2} \frac{1}{\kappa^{2}} \gamma_{2}\right] \frac{d^{2} W}{d \eta^{2}}\right]+\chi \beta \frac{\bar{h}^{2}}{24}(1-v) \frac{\partial \varphi_{m}}{\partial \eta}, \\
\left(\kappa^{2}-\gamma_{1} \lambda^{2} \beta \frac{\chi^{4} \bar{h}^{4}}{144}\right) \psi_{y} & \\
= & -\chi \frac{\partial}{\partial \eta}\left[\left[\frac{\beta^{2} \chi^{4} \bar{h}^{4}}{144} \lambda^{2} \frac{1}{\kappa^{2}}\left(1+(m \pi)^{2} \gamma_{2} \frac{\bar{h}^{2}}{12}\right)-(m \pi)^{2} \frac{\beta \bar{h}^{2}}{12}+\kappa^{2}-\gamma_{3} \lambda^{2} \beta \frac{\chi^{4} \bar{h}^{4}}{144}\right] W\right. \\
& \left.+\left[\chi^{2} \frac{\beta \bar{h}^{2}}{12}-\frac{\beta^{2} \chi^{6} \bar{h}^{6}}{1728} \lambda^{2} \frac{1}{\kappa^{2}} \gamma_{2}\right] \frac{d^{2} W}{d \eta^{2}}\right]+m \pi \beta \frac{\bar{h}^{2}}{24}(1-v) \varphi_{m} .
\end{aligned}
$$

The roots of the characteristic polynomial of Eq. (50) are:

$$
r_{ \pm}^{2}=\frac{-\Lambda_{2} \pm \sqrt{\Lambda_{2}^{2}-4 \Lambda_{1} \Lambda_{3}}}{2 \Lambda_{1}} .
$$

$r_{-}^{2}$ and $r_{+}^{2}$ are both equal to $(m \pi / \alpha)^{2}$, positive number, for $\lambda$ equal to 0 . There is a transition frequency, denoted $\lambda_{-}^{*}$, such that for $\lambda$ greater than $\lambda_{-}^{*}, r_{-}^{2}$ becomes negative, and so $r_{-}$is an imaginary number. Similarly, when $\lambda$ is greater than a value $\lambda_{+}^{*}, r_{+}$is an imaginary number. For instance, the values of $\lambda_{-}^{*}$ and $\lambda_{+}^{*}$ are listed in the following table for the eight first values of $m$, with an aspect ratio $\alpha$, a thickness ratio $\bar{h}$, a shear correction factor $\kappa^{2}$, and a Poisson ratio $v$ equal to $1,0.2,0.86667$, and 0.3 , respectively, for the three versions of the Uflyand-Mindlin plate theory. For the particular case of the truncated Uflyand-Mindlin plate model, one can show that $\lambda_{+}^{*}$ go to infinity. Thus, $r_{+}$is always real, independent of the frequency.

The table shows that there is zero or one unique root for any model: The sign of $\lambda_{-}^{*}$ and $\lambda_{+}^{*}$ changes only once. Moreover, $\lambda_{-}^{*}$ is smaller than $\lambda_{+}^{*} . \lambda_{-}^{*}$ and $\lambda_{+}^{*}$ are reached for a certain value of the order of the natural frequency. This value depends on the model, the boundary conditions, and the geometric parameters such as the thickness ratio. For instance, for a plate with all edges simply supported, as shown in Table 2, keeping the same set of parameters in Table $1\left(\kappa^{2} ; v ; \alpha ; \bar{h}\right)=(0.86667 ; 0.3 ; 1 ; 0.2), \lambda$ is immediately greater than $\lambda_{-}^{*}$, and it is greater than $\lambda_{-}^{*}$ after the 18th and the 34th natural frequency for original Uflyand-Mindlin plate theory and the Uflyand-Mindlin plate model based on slope inertia, respectively. It is worth noting that, for this particular case, considering the original Uflyand-Mindlin plate theory, the second branch of frequencies and the transition to the frequency greater than $\lambda_{+}^{*}$ occur at the same moment. Thus, the second transition frequency $\lambda_{+}^{*}$ is reached for big orders of frequencies, not considered usually in the literature.

Thus, we consider the different models and different cases. The solution of the differential equation depends on the value of the frequency $\lambda$. 
Table 1 Eight first values of the transition frequencies $\lambda_{-}^{*}$ and $\lambda_{+}^{*}$ for the three versions of the Uflyand-Mindlin plate model with $\left(\kappa^{2} ; v ; \chi ; \bar{h}\right)=(0.86667 ; 0.3 ; 1 ; 0.2)$

\begin{tabular}{|c|c|c|c|c|c|c|c|c|c|}
\hline & $\mathrm{m}$ & 1 & 2 & 3 & 4 & 5 & 6 & 7 & 8 \\
\hline Original & $\lambda_{-}^{*}$ & 9.25 & 31.94 & 60.76 & 91.91 & 123.79 & 155.82 & 187.74 & 219.487 \\
\hline & $\lambda_{+}^{*}$ & 176.27 & 204.24 & 241.53 & 283.89 & 329.32 & 376.76 & 425.61 & 475.50 \\
\hline Truncated & $\lambda_{-}^{*}$ & 9.24 & 31.55 & 58.93 & 87.44 & 115.88 & 143.99 & 171.77 & 199.28 \\
\hline & $\lambda_{+}^{*}$ & Infinite & & & & & & & \\
\hline Slope inertia & $\lambda_{-}^{*}$ & $\begin{array}{r}9.21 \\
1652\end{array}$ & 30.46 & $\begin{array}{r}52.61 \\
\end{array}$ & $\begin{array}{r}70.00 \\
\end{array}$ & $\begin{array}{r}82.27 \\
\end{array}$ & 90.73 & 96.61 & 100.79 \\
\hline
\end{tabular}

If $\lambda \leq \lambda_{-}^{*}<\lambda_{+}^{*}$ then $r_{-}^{2} \geq 0 ; r_{+}^{2}>0$. The solution of the differential equation is given by

$$
W_{m}(\eta)=C_{1} \cosh r_{+} \eta+C_{2} \sinh r_{+} \eta+C_{3} \cosh r_{-} \eta+C_{4} \sinh r_{-} \eta .
$$

Define $\tilde{r}_{-}$and $\tilde{r}_{+}$as follows:

$$
\tilde{r}_{-}^{2}=\frac{\Lambda_{2}+\sqrt{\Lambda_{2}^{2}-4 \Lambda_{1} \Lambda_{3}}}{2 \Lambda_{1}} ; \quad \tilde{r}_{+}^{2}=\frac{\Lambda_{2}-\sqrt{\Lambda_{2}^{2}-4 \Lambda_{1} \Lambda_{3}}}{2 \Lambda_{1}} .
$$

If $\lambda_{-}^{*}<\lambda \leq \lambda_{+}^{*}$ then $r_{-}^{2}<0 ; r_{+}^{2} \geq 0$; and $\cosh r_{-} \eta$ and $\sinh r_{-} \eta$ are replaced by $\cos \tilde{r}_{-} \eta$ and $\sin \tilde{r}_{-} \eta$, respectively. If $\lambda_{-}^{*}<\lambda ; \lambda_{+}^{*}<\lambda$ then, in addition to the previously given transformation, $\cosh r_{+} \eta$ and $\sinh r_{+} \eta$ are replaced by $\cos \tilde{r}_{-} \eta$ and $\sin \tilde{r}_{-} \eta$, respectively.

This last case does not appear in the paper in the paper of Hashemi and Arsanjani [26]. Indeed, he determines the first values of the natural frequencies. Thus, the frequency is always smaller than the second transition value $\lambda_{+}^{*}$.

Thus, the process to determine the natural frequency is the following. First of all, for fixed values of the aspect ratio $\alpha$, the thickness ratio $\bar{h}$, the mode shape number $m$, the shear coefficient $\kappa^{2}$, and the Poisson's ratio $\nu$, the solution $\lambda$ is obtained by considering all the possible expressions of the displacements given by Eq. (56).

The roots of the characteristic equation Eq. (51) are:

$$
u_{ \pm}= \pm \sqrt{\frac{\Lambda_{5}}{\Lambda_{4}}}
$$

hence, the solution is expressed as follows:

$$
\varphi_{m}(\eta)=C_{5} \sinh u_{+} \eta+C_{6} \cosh u_{+} \eta .
$$

This expression coincides with the result obtained by Jomehzadeh and Saidi [32].

In the following, three different boundary conditions are studied: simply supported, free, and clamped (fixed) (see, for instance, Wang et al. [33]).

Clamped edges:

$$
w=0 ; \quad \psi_{x}=0 ; \quad \psi_{y}=0
$$

Simply supported:

$$
w=0 ; \quad \frac{\partial \psi_{y}}{\partial y}+v \frac{\partial \psi_{x}}{\partial x}=0 ; \quad \psi_{x}=0
$$

Free edges:

$$
\kappa^{2} G h\left(\psi_{y}+\frac{\partial w}{\partial y}\right)-\gamma_{2} \frac{\rho h^{3}}{12} \omega^{2} \frac{\partial w}{\partial y}=0 ; \quad \frac{\partial \psi_{y}}{\partial y}+v \frac{\partial \psi_{x}}{\partial x}=0 ; \quad \frac{1}{2}(1-v) D\left(\frac{\partial \psi_{x}}{\partial y}+\frac{\partial \psi_{y}}{\partial x}\right)=0
$$




\section{Numerical results}

6.1 Two opposite edges simply supported and two other edges both clamped or free

First of all, for a thin plate, the non-dimensional natural frequencies are the solutions of characteristic equations given by Leissa [19] and Wang and Wang [36]. Following notations are introduced:

$$
\lambda_{1}=\frac{1}{b} \sqrt{(m \pi)^{2}+\lambda^{2}} ; \quad \lambda_{2}=\frac{1}{b} \sqrt{(m \pi)^{2}-\lambda^{2}} ; \quad \tilde{\lambda}_{2}=\frac{1}{b} \sqrt{\lambda^{2}-(m \pi)^{2}} .
$$

Thus, for an SCSC plate,

For $m \pi<\lambda$

$$
2 \lambda_{1} \tilde{\lambda}_{2}\left[1-\cosh \frac{\lambda_{1}}{\chi} \cos \frac{\tilde{\lambda}_{2}}{\chi}\right]+\left(\lambda_{1}^{2}-\tilde{\lambda}_{2}^{2}\right) \sinh \frac{\lambda_{1}}{\chi} \sin \frac{\tilde{\lambda}_{2}}{\chi}=0
$$

If $\lambda \leq m \pi$ then $\tilde{\lambda}_{2}, \cos \left(\tilde{\lambda}_{2} / \chi\right)$, and $\sin \left(\tilde{\lambda}_{2} / \chi\right)$ are replaced by $\lambda_{2}, \cosh \left(\lambda_{2} / \chi\right)$, and $\sinh \left(\lambda_{2} / \chi\right)$, respectively. For an SFSF plate, for $m \pi<\lambda$

$$
\begin{aligned}
& \left\{\lambda_{1}^{2}\left[k-(1-v)(m \pi)^{2}\right]^{4}-\tilde{\lambda}_{2}^{2}\left[k+(1-v)(m \pi)^{2}\right]^{4}\right\} \sinh \frac{\lambda_{1}}{\chi} \sin \frac{\tilde{\lambda}_{2}}{\chi} \\
& +2 \lambda_{1} \tilde{\lambda}_{2}\left[\left(\lambda_{1} \tilde{\lambda}_{2}\right)^{2}-v(1-v)(m \pi)^{4}\right]\left(1-\cosh \frac{\lambda_{1}}{\chi} \cos \frac{\tilde{\lambda}_{2}}{\chi}\right)=0 .
\end{aligned}
$$

If $\lambda \leq m \pi$ then $\tilde{\lambda}_{2}, \cos \left(\tilde{\lambda}_{2} / \chi\right)$, and $\sin \left(\tilde{\lambda}_{2} / \chi\right)$ are replaced by $\lambda_{2}, \cosh \left(\lambda_{2} / \chi\right)$, and $\sinh \left(-\lambda_{2} / \chi\right)$, respectively.

For a thick plate, when two opposite edges have the same boundary conditions (SFSF and SCSC), because of the symmetry of the problem, it is suspected that all possible modes of vibration will either be symmetric or antisymmetric with respect to the central axis and, consequently, the $\xi$ axis is arbitrarily located at the center of the plate. Two cases are considered: symmetric and antisymmetric modes. In contrast to the simply supported case, the coefficients $C_{1}, C_{3}$, and $C_{4}$ are not both equal to 0 and the wave forms are only approximately sinusoidal in the $y$ direction. Moreover, defining the node lines as the lines across which the displacements vanish [26], the node lines in the $y$ direction will be parallel to the $y$ axis. For two opposite edges both clamped or free, the node lines will be also parallel to the $x$ axis. The edges $\xi=0$ and $\xi=1$ are simply supported. As explained by Leissa [19], for the particular case and in the $y$ direction, the wave forms are found to be sine function exactly.

Symmetric modes Restricting to this family of modes, the antisymmetric terms are deleted from the previous equations. The boundary conditions are applied at the edges $\eta=-1 / 2$ and $\eta=1 / 2$. Because $\partial w / \partial y$ is a function of $\psi_{y}$, when the symmetric modes for $w$ are retained, only the antisymmetric modes are kept from the expression of $\psi_{y}$. According to Eqs. (37) and (38), $\varphi_{m}$ can be expressed with only $\psi_{y}$ and the derivative following $x$ of $w$, both being antisymmetric. Consequently, only the antisymmetric term of $\varphi_{m}$ is retained. The displacement is expressed as:

For $\lambda \leq \lambda_{-}^{*}<\lambda_{+}^{*}$ then $r_{-}^{2} \geq 0 ; r_{+}^{2}>0$. The solution of the differential equation is given by

$$
W_{m}(\eta)=C_{1} \cosh r_{+} \eta+C_{3} \cosh r_{-} \eta \text {. }
$$

If $\lambda_{-}^{*}<\lambda \leq \lambda_{+}^{*}$ then $r_{-}^{2}<0 ; r_{+}^{2} \geq 0$ and $\cosh r_{-} \eta$ is replaced by $\cos \tilde{r}_{-} \eta$. If $\lambda_{-}^{*}<\lambda ; \lambda_{+}^{*}<\lambda$ then $\cosh r_{-} \eta$ and $\cosh r_{+} \eta$ are replaced by $\cos \tilde{r}_{-} \eta$ and $\cos \tilde{r}_{-} \eta$, respectively. Moreover, $\varphi_{m}$ is given by

$$
\varphi_{m}(y)=C_{5} \sinh u_{+} \eta
$$

Applying the boundary conditions, one obtains a system of three equations with three unknowns, which can be written in matrix form:

$$
Z_{S}\left(\begin{array}{l}
C_{1} \\
C_{3} \\
C_{5}
\end{array}\right)=\left(\begin{array}{l}
0 \\
0 \\
0
\end{array}\right)
$$


where $Z_{S}$ is a matrix $3 \times 3$. In order to have a non-trivial solution, the determinant of $Z_{S}$ must vanish and it provides the natural frequencies of the plate.

The symmetric modes have an axis of symmetry with respect to the $y$ coordinate. Thus, $(m, n)=$ $(1,1),(m, n)=(2,1) ;(m, n)=(3,1)$ or $(m, n)=(3,1)$ are symmetric [26].

Antisymmetric modes By the same way, the symmetric terms are deleted from the previous equations. The boundary conditions are applied at the edges $\eta=-1 / 2$ and $\eta=1 / 2$. Only the symmetric term of $\varphi_{m}$ is kept. The displacement is expressed as:

- $\lambda \leq \lambda_{-}^{*}<\lambda_{+}^{*}$ then $r_{-}^{2} \geq 0 ; r_{+}^{2}>0$.

$$
W_{m}(\eta)=C_{2} \sinh r_{+} \eta+C_{4} \sinh r_{-} \eta
$$

- If $\lambda_{-}^{*}<\lambda \leq \lambda_{+}^{*}$ then $\sinh r_{-} \eta$ is replaced by $\sin \tilde{r}_{-} \eta$. If $\lambda_{-}^{*}<\lambda$ then $\sinh r_{-} \eta$ and $\sinh r_{+} \eta$ are replaced by $\sin \tilde{r}_{-} \eta$ and $\sin \tilde{r}_{-} \eta$, respectively. Moreover, $\varphi_{m}$ is given by

$$
\varphi_{m}(y)=C_{6} \cosh u_{+} \eta
$$

Applying the boundary conditions, one obtains a system of three equations with three unknowns, which is written in matrix form:

$$
Z_{A S}\left(\begin{array}{l}
C_{2} \\
C_{4} \\
C_{6}
\end{array}\right)=\left(\begin{array}{l}
0 \\
0 \\
0
\end{array}\right),
$$

where $Z_{A S}$ is a matrix $3 \times 3$. In order to have a non-trivial solution, the determinant of $Z_{A S}$ must vanish and it provides the natural frequencies of the plate.

The antisymmetric modes have an axis of symmetry with respect to the $y$ coordinate. Thus, $(m, n)=$ $(1,2),(m, n)=(2,2) ;(m, n)=(3,2)$ or $(m, n)=(4,2)$ are antisymmetric [26].

By considering separately the symmetric and the antisymmetric modes for the case of a plate with two simply supported opposite edges and the two other opposite edges having the same boundary conditions, Leissa [23] and Gorman [24,25] reduce the problem to the calculation of values of frequency for which the determinant vanishes. Consequently, the time to calculate the determinant is considerably reduced. Indeed, it is much easier and efficient to calculate two determinants of matrices $3 \times 3$ (total of 18 coefficients) than one determinant of a matrix $6 \times 6$ (total of 36 coefficients).

First of all, three functions $H_{1}, H_{2}$, and $H_{3}$ are defined such as

$$
\begin{gathered}
H_{1}(x)=\frac{\beta^{2} \chi^{4} \bar{h}^{4}}{144} \frac{\lambda^{2}}{\kappa^{2}}-\left[\chi^{2} x+(m \pi)^{2}\right] \frac{\beta \bar{h}^{2}}{12}\left(1-\gamma_{2} \lambda^{2} \bar{h}^{4} \chi^{4} \frac{\beta}{144 \kappa^{2}}\right)+\kappa^{2}-\gamma_{3} \lambda^{2} \beta \frac{\chi^{4} \bar{h}^{4}}{144}, \\
H_{2}(x)=\chi\left(\kappa^{2}-\left[\gamma_{1}+\gamma_{2}\right] \frac{\beta \bar{h}^{4} \chi^{4}}{144} \lambda^{2}-H_{1}(x)\right), \\
H_{3}(x)=\left[\chi^{2} x+v(m \pi)^{2}\right] .
\end{gathered}
$$

The following notations are also considered:

$$
\begin{aligned}
H_{i+} & =H_{i}\left(-r_{+}^{2}\right) ; \quad H_{i \tilde{+}}=H_{i}\left(\tilde{r}_{+}^{2}\right) ; \quad H_{i-}=H_{i}\left(-r_{-}^{2}\right) ; \quad H_{i \tilde{+}}=H_{i}\left(\tilde{r}_{-}^{2}\right) \quad(i=1,2,3), \\
C_{x} & =\cosh x ; \quad S_{x}=\sinh x ; \quad \tilde{C}_{x}=\cos \tilde{x} ; \quad \tilde{S}_{x}=\sin \tilde{x} ; \quad \bar{\beta}=(1-v) \frac{\beta \bar{h}^{2}}{24} .
\end{aligned}
$$

For the particular case of a plate with two opposite edges that are clamped, the matrices $Z_{S}$ and $Z_{A S}$ are expressed as follows:

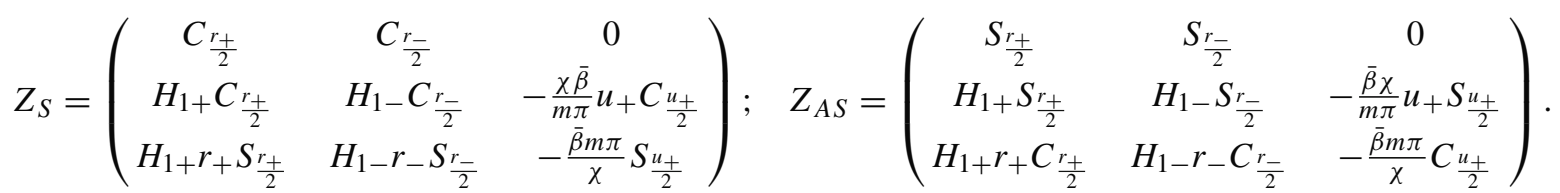


For the particular case of a plate with two opposite edges that are free, the matrices $Z_{S}$ and $Z_{A S}$ are expressed as follows:

$$
\begin{aligned}
& Z_{S}=\left(\begin{array}{ccc}
H_{3+} H_{1+} C_{\frac{r_{+}}{2}} & H_{3-} H_{1-} C_{\frac{r_{-}}{2}} & \bar{\beta}(1-v) \chi m \pi u_{+} C_{\frac{u_{+}}{2}} \\
H_{2+} r_{+} S_{\frac{r_{+}}{2}} & H_{2-} r_{-} S_{\frac{r_{-}}{2}} & m \pi \bar{\beta} S_{\frac{u_{+}}{2}} \\
m \pi \chi H_{1+} r_{+} S_{\frac{r_{+}}{2}} & m \pi \chi H_{1-} r_{-} S_{\frac{r_{-}}{2}} & -\frac{\bar{\beta}}{2}\left[\chi^{2} u_{+}^{2}+(m \pi)^{2}\right] S_{\frac{u_{+}}{2}}
\end{array}\right), \\
& Z_{A S}=\left(\begin{array}{ccc}
H_{3+} H_{1+} S_{\frac{r_{+}}{2}} & H_{3-} H_{1-} S_{\frac{r_{-}}{2}} & \bar{\beta}(1-v) \chi m \pi u_{+} S_{\frac{u_{+}}{2}} \\
H_{2+} r_{+} C_{\frac{r_{+}}{2}} & H_{2-} r_{-} C_{\frac{r_{-}}{2}} & m \pi \bar{\beta} C_{\frac{u_{+}}{2}} \\
m \pi \chi H_{1+} r_{+} C_{\frac{r_{+}}{2}} & m \pi \chi H_{1-} r_{-} C_{\frac{r_{-}}{2}} & -\frac{\bar{\beta}}{2}(1-v)\left[\chi^{2} u_{+}^{2}+(m \pi)^{2}\right] C_{\frac{u_{+}}{2}}
\end{array}\right) .
\end{aligned}
$$

In both cases, if $\lambda_{-}^{*}<\lambda \leq \lambda_{+}^{*}$ then $r_{-}^{2}<0 ; r_{+}^{2} \geq 0,-r_{-}^{2}, C_{\frac{r_{-}}{2}} ; r_{-} S_{\frac{r_{-}}{2}} ; S_{\frac{r_{-}}{2}}$ and $r_{-} C_{\frac{r_{-}}{2}}$ are replaced by $\tilde{r}_{-}^{2}, \tilde{C}_{\frac{r_{-}}{2}},-\tilde{r}_{-} \tilde{S}_{\frac{r_{-}}{2}}, \tilde{S}_{\frac{r_{-}}{2}}$ and $\tilde{r}_{-} \tilde{C}_{\frac{r_{-}}{2}}$, respectively. If $\lambda_{-}^{*}<\lambda ; \lambda_{+}^{*}<\lambda$ then $r_{-}^{2}<0 ; r_{+}^{2}<0,-r_{+}^{2}, C_{\frac{r_{+}}{2}}$; $r_{-} S_{\frac{r_{+}}{2}} ; \quad S_{\frac{r_{+}}{2}}$ and $r_{+} C_{\frac{r_{+}}{2}}$ are replaced by $\tilde{r}_{-}^{2}, \tilde{C}_{\frac{r_{+}}{2}},-\tilde{r}_{+} \tilde{S}_{\frac{r_{+}}{2}}, \tilde{S}_{\frac{r_{+}}{2}}$ and $\tilde{r}_{+} \tilde{C}_{\frac{r_{+}}{2}}$, respectively.

\subsection{Two other opposite edges with differing boundary conditions}

When two opposite edges have differing boundary conditions (SSSF, SSSC, and SCSF), the problem cannot be separated in symmetric and antisymmetric modes. The $\xi$ axis is arbitrarily located at one of the edges of the plate. Thus, the boundary conditions are applied at the edges $\eta=0$ and $\eta=1$. In contrast to the case of two opposite edges having the same boundary conditions, the node lines are not straight or parallel to the $x$ axis. Applying the boundary conditions, one obtains a system of six equations with six unknowns, which is in matrix form:

$$
Z\left(\begin{array}{l}
C_{1} \\
C_{2} \\
C_{3} \\
C_{4} \\
C_{5} \\
C_{6}
\end{array}\right)=\left(\begin{array}{l}
0 \\
0 \\
0 \\
0 \\
0 \\
0
\end{array}\right),
$$

where $Z$ is a matrix $6 \times 6$. In order to have a non-trivial solution, the determinant of $Z$ must vanish and it provides the natural frequencies of the plate.

For the particular case of a plate with the edge $\eta=0$ free and the edge $\eta=1$ simply supported (SSSF plate), the coefficients $Z_{i j}(i=1,2,3,4,5,6)$ for the matrix $Z$ are

$\bullet \lambda \leq \lambda_{-}^{*}<\lambda_{+}^{*}$ then $r_{-}^{2} \geq 0 ; r_{+}^{2}>0$.

$$
\begin{aligned}
& Z_{12}=H_{3+} H_{1+} ; Z_{14}=H_{3-} H_{1-} ; Z_{16}=\chi \bar{\beta}(1-v) m \pi u_{+} ; Z_{21}=H_{2+} r_{+} ; Z_{23}=H_{2-} r_{-} ; Z_{25}=\bar{\beta} m \pi ; \\
& Z_{31}=-2 \chi m \pi r_{+} H_{1+} ; Z_{33}=-2 \chi m \pi r_{-} H_{1-} ; Z_{35}=\bar{\beta}\left[\chi^{2} u_{+}^{2}+(m \pi)^{2}\right] ; Z_{41}=S_{r_{+}} ; Z_{42}=C_{r_{+}} ; \\
& Z_{43}=S_{r_{-}} ; Z_{44}=C_{r_{-}} ; Z_{51}=H_{3+} H_{1+} S_{r_{+}} ; Z_{52}=H_{3+} H_{1+} C_{r_{+}} ; Z_{53}=H_{3-} H_{1-} S_{r_{-}} ; Z_{54}=H_{3-} H_{1-} C_{r_{-}} \\
& Z_{55}=\bar{\beta}(1-v) \chi m \pi u_{+} S_{u_{+}} ; Z_{56}=\bar{\beta}(1-v) \chi m \pi u_{+} C_{u_{+}} ; Z_{61}=H_{1+} S_{r_{+}} ; Z_{62}=H_{1+} C_{r_{+}} ; \\
& Z_{63}=H_{1-} S_{r_{-}} ; Z_{64}=H_{1-} C_{r_{-}} ; Z_{65}=-\frac{\bar{\beta} \chi}{m \pi} S_{u_{+}} ; Z_{66}=\frac{\bar{\beta} \chi}{m \pi} C_{u_{+}} ; \\
& Z_{11}=Z_{13}=Z_{15}=Z_{22}=Z_{24}=Z_{26}=Z_{32}=Z_{34}=Z_{36}=Z_{45}=Z_{46}=0
\end{aligned}
$$

$\bullet \lambda_{-}^{*}<\lambda \leq \lambda_{+}^{*}$ then $r_{-}^{2}<0 ; r_{+}^{2} \geq 0$ and the coefficients $\tilde{Z}_{14}, \tilde{Z}_{23}, \tilde{Z}_{33}, \tilde{Z}_{43}, \tilde{Z}_{44}, \tilde{Z}_{53}, \tilde{Z}_{54}, \tilde{Z}_{63}$ and $\tilde{Z}_{64}$ are replaced by

$$
\begin{aligned}
& Z_{14}=\tilde{H}_{3-} \tilde{H}_{1-} ; Z_{23}=\tilde{H}_{2-} \tilde{r}_{-} ; Z_{33}=-2 m \pi \chi \tilde{H}_{1-} \tilde{r}_{-} ; Z_{43}=\tilde{S}_{r_{-}} ; Z_{44}=\tilde{C}_{r_{-}} \\
& Z_{53}=\tilde{H}_{3-} \tilde{H}_{1-} \tilde{S}_{r_{-}} ; Z_{54}=\tilde{H}_{3-} \tilde{H}_{1-} \tilde{C}_{r_{-}} ; Z_{63}=\tilde{H}_{1-} \tilde{S}_{r_{-}} ; Z_{64}=\tilde{H}_{1-} \tilde{C}_{r_{-}}
\end{aligned}
$$


- $\lambda_{-}^{*}<\lambda ; \lambda_{+}^{*}<\lambda$ then $r_{-}^{2}<0 ; r_{+}^{2}<0$ and matrix $\mathrm{Z}$ is given by the coefficients $\tilde{Z}_{i j}$ with $\tilde{Z}_{14}, \tilde{Z}_{23}, \tilde{Z}_{33}, \tilde{Z}_{43}$, $\tilde{Z}_{44}, \tilde{Z}_{53}, \tilde{Z}_{54}, \tilde{Z}_{63}$ and $\tilde{Z}_{64}$ given in the previous equations and the other coefficients given by

$$
\begin{aligned}
& \tilde{Z}_{16}=Z_{16} ; \tilde{Z}_{25}=Z_{25} ; \tilde{Z}_{35}=Z_{35} ; \tilde{Z}_{55}=Z_{55} ; Z_{56}=\tilde{Z}_{56} ; \tilde{Z}_{65}=Z_{65} ; \tilde{Z}_{66}=Z_{66} \\
& Z_{12}=\tilde{H}_{3+} \tilde{H}_{1+} ; Z_{21}=\tilde{H}_{2+} \tilde{r}_{+} ; Z_{31}=-2 m \pi \chi \tilde{H}_{1+} \tilde{r}_{+} ; Z_{41}=\tilde{S}_{r_{+}} ; \\
& Z_{42}=\tilde{C}_{r_{+}} ; Z_{51}=\tilde{H}_{3+} \tilde{H}_{1+} \tilde{S}_{r_{+}} ; Z_{52}=\tilde{H}_{3+} \tilde{H}_{1+} \tilde{C}_{r_{+}} ; Z_{61}=\tilde{r}_{+} \tilde{H}_{1+} \tilde{S}_{r_{+}} ; Z_{62}=\tilde{r}_{+} \tilde{H}_{1+} \tilde{C}_{r_{+}} ; \\
& Z_{11}=Z_{13}=Z_{15}=Z_{22}=Z_{24}=Z_{26}=Z_{32}=Z_{34}=Z_{36}=Z_{45}=Z_{46}=0
\end{aligned}
$$

Moreover, for a thin plate, the frequencies are obtained through the following characteristic equation [19],

- $m \pi<\lambda$

$$
\tilde{\lambda}_{2}\left[\lambda+(1-v)(m \pi)^{2}\right]^{2} \sinh \frac{\lambda_{1}}{\chi} \cos \frac{\tilde{\lambda}_{2}}{\chi}-\lambda_{1}\left[k-(1-v)(m \pi)^{2}\right]^{2} \cosh \frac{\lambda_{1}}{\chi} \sin \frac{\tilde{\lambda}_{2}}{\chi}=0
$$

For $\lambda \leq m \pi, \tilde{\lambda}_{2}, \sin \left(\tilde{\lambda}_{2} / \chi\right)$, and $\cos \left(\tilde{\lambda}_{2} / \chi\right)$ are replaced by $\lambda_{2}, \sin \left(\lambda_{2} / \chi\right)$, and $\cos \left(\lambda_{2} / \chi\right)$, respectively.

For the particular case of a plate with the edge $\eta=0$ simply supported and the edge $\eta=1$ clamped (SSSC plate), the coefficients $Z_{i j}(i=1,2,3,4,5,6)$ for the matrix $Z$ are

$\bullet \lambda \leq \lambda_{-}^{*}<\lambda_{+}^{*}$ then $r_{-}^{2} \geq 0 ; r_{+}^{2}>0$.

$$
\begin{aligned}
& Z_{12}=Z_{14}=1 ; Z_{16}=Z_{11}=Z_{13}=Z_{15}=Z_{21}=Z_{23}=Z_{25}=Z_{32}=Z_{34}=Z_{36}=Z_{45}=Z_{46}=0 \\
& Z_{22}=H_{3+} H_{1+} ; Z_{24}=H_{3-} H_{1-} ; Z_{26}=\bar{\beta}(1-v) \chi m \pi u_{+} ; Z_{32}=H_{1+} ; Z_{34}=H_{1-} ; Z_{36}=-\bar{\beta} \frac{m \pi}{\chi} ; \\
& Z_{41}=S_{r_{+}} ; Z_{42}=C_{r_{+}} ; Z_{43}=S_{r_{-}} ; Z_{44}=C_{r_{-}} ; Z_{51}=H_{1+} S_{r_{+}} ; Z_{52}=H_{1+} C_{r_{+}} ; Z_{53}=H_{1-} S_{r_{-}} ; \\
& Z_{54}=H_{1-} C_{r_{-}} ; Z_{55}=-\frac{\bar{\beta} \chi}{m \pi} u_{+} S_{u_{+}} ; Z_{56}=-\frac{\bar{\beta} \chi}{m \pi} u_{+} C_{u_{+}} ; Z_{61}=H_{1+} r_{+} C_{r_{+}} ; Z_{62}=H_{1+} r_{+} S_{r_{+}} ; \\
& Z_{63}=H_{1-} r_{-} C_{r_{-}} ; Z_{64}=H_{1-} r_{-} S_{r_{-}} ; Z_{65}=-\bar{\beta} \frac{m \pi}{\chi} C_{u_{+}} ; Z_{66}=-\bar{\beta} \frac{m \pi}{\chi} S_{u_{+}}
\end{aligned}
$$

- $\lambda_{-}^{*}<\lambda \leq \lambda_{+}^{*}$ then $r_{-}^{2}<0 ; r_{+}^{2} \geq 0$ and the coefficients $\tilde{Z}_{24}, \tilde{Z}_{34}, \tilde{Z}_{43}, \tilde{Z}_{44}, \tilde{Z}_{53}, \tilde{Z}_{54}, \tilde{Z}_{63}$ and $\tilde{Z}_{64}$ are replaced by

$$
\begin{aligned}
& Z_{24}=\tilde{H}_{3-} \tilde{H}_{1-} ; Z_{34}=\tilde{H}_{1-} ; Z_{43}=\tilde{S}_{r_{-}} ; Z_{44}=\tilde{C}_{r_{-}} ; \\
& Z_{53}=\tilde{H}_{1-} \tilde{S}_{r_{-}} ; Z_{54}=\tilde{H}_{1-} \tilde{C}_{r_{-}} ; Z_{63}=\tilde{H}_{1-} \tilde{r}_{-} \tilde{C}_{r_{-}} ; Z_{64}=-\tilde{H}_{1-} \tilde{r}_{-} \tilde{S}_{r_{-}}
\end{aligned}
$$

- $\lambda_{-}^{*}<\lambda ; \lambda_{+}^{*}<\lambda$ then $r_{-}^{2}<0 ; r_{+}^{2}<0$, and matrix $Z$ is given by the coefficients $\tilde{Z}_{i j}$ with $\tilde{Z}_{24}, \tilde{Z}_{34}, \tilde{Z}_{43}$, $\tilde{Z}_{44}, \tilde{Z}_{53}, \tilde{Z}_{54}, \tilde{Z}_{63}$ and $\tilde{Z}_{64}$ given in the previous equations and the other coefficients given by

$$
\begin{aligned}
& \tilde{Z}_{26}=Z_{26} ; \tilde{Z}_{36}=Z_{36} ; \tilde{Z}_{55}=Z_{55} ; \tilde{Z}_{56}=Z_{56} ; \tilde{Z}_{65}=Z_{65} ; \tilde{Z}_{66}=Z_{66} \\
& Z_{22}=\tilde{H}_{3+} \tilde{H}_{1+} ; Z_{32}=\tilde{H}_{1+} ; \\
& Z_{41}=\tilde{S}_{r_{+}} ; Z_{42}=\tilde{C}_{r_{+}} ; Z_{51}=\tilde{H}_{1+} \tilde{S}_{r_{+}} ; Z_{52}=\tilde{H}_{1+} \tilde{C}_{r_{+}} ; Z_{61}=\tilde{H}_{1+} \tilde{r}_{+} \tilde{C}_{r_{+}} ; Z_{62}=-\tilde{H}_{1+} \tilde{r}_{+} \tilde{S}_{r_{+}} ; \\
& Z_{12}=Z_{14}=1 ; Z_{16}=Z_{11}=Z_{13}=Z_{15}=Z_{21}=Z_{23}=Z_{25}=Z_{32}=Z_{34}=Z_{36}=Z_{45}=Z_{46}=0
\end{aligned}
$$

Moreover, for a thin plate, the frequencies are obtained through the following characteristic equation [19],

- $m \pi<\lambda$

$$
\lambda_{1} \cosh \frac{\lambda_{1}}{\chi} \sin \frac{\tilde{\lambda}_{2}}{\chi}-\tilde{\lambda}_{2} \sinh \frac{\lambda_{1}}{\chi} \cos \frac{\tilde{\lambda}_{2}}{\chi}=0 .
$$

For $\lambda \leq m \pi, \tilde{\lambda}_{2}, \sin \left(\tilde{\lambda}_{2} / \chi\right)$, and $\cos \left(\tilde{\lambda}_{2} / \chi\right)$ are replaced by $\lambda_{2}, \sin \left(\lambda_{2} / \chi\right)$, and $\cos \left(\lambda_{2} / \chi\right)$, respectively. For the particular case of a plate with the edge $\eta=0$ free and the edge $\eta=1$ clamped (SCSF plate), the coefficients $Z_{i j}(i=1,2,3,4,5,6)$ for the matrix $Z$ are 


$$
\bullet \lambda \leq \lambda_{-}^{*}<\lambda_{+}^{*} \text { then } r_{-}^{2} \geq 0 ; r_{+}^{2}>0
$$$$
Z_{12}=H_{3+} H_{1+} ; Z_{14}=H_{3-} H_{1-} ; Z_{16}=\chi \bar{\beta}(1-v) m \pi u_{+} ; Z_{21}=H_{2+} r_{+} ; Z_{23}=H_{2-} r_{-} ; Z_{25}=m \pi \bar{\beta} ;
$$$$
Z_{31}=-2 \chi m \pi H_{1+} r_{+} ; Z_{33}=-2 \chi m \pi H_{1-} r_{-} ; Z_{35}=\bar{\beta}\left[\chi^{2} u_{+}^{2}+(m \pi)^{2}\right] ; Z_{41}=S_{r_{+}} ; Z_{42}=C_{r_{+}} \text {; }
$$$$
Z_{43}=S_{r_{-}} ; Z_{44}=C_{r_{-}} ; Z_{51}=H_{1+} S_{r_{+}} ; Z_{52}=H_{1+} C_{r_{+}} ; Z_{53}=H_{1-} S_{r_{-}} ; Z_{54}=H_{1-} C_{r_{-}} \text {; }
$$$$
Z_{55}=-\frac{\bar{\beta} \chi}{m \pi} u_{+} S_{u_{+}} ; Z_{56}=-\frac{\bar{\beta} \chi}{m \pi} u_{+} C_{u_{+}} ; Z_{61}=H_{1+} r_{+} C_{r_{+}} ; Z_{62}=H_{1+} r_{+} S_{r_{+}} ;
$$$$
Z_{63}=H_{1-} r_{-} C_{r_{-}} ; Z_{64}=H_{1-} r_{-} S_{r_{-}} ; Z_{65}=-\bar{\beta} \frac{m \pi}{\chi} C_{u_{+}} ; Z_{66}=-\bar{\beta} \frac{m \pi}{\chi} S_{u_{+}}
$$$$
Z_{11}=Z_{13}=Z_{15}=Z_{22}=Z_{24}=Z_{26}=Z_{32}=Z_{34}=Z_{36}=Z_{45}=Z_{46}=0
$$

- $\lambda_{-}^{*}<\lambda \leq \lambda_{+}^{*}$ then $r_{-}^{2}<0 ; r_{+}^{2} \geq 0$ and the coefficients $\tilde{Z}_{14}, \tilde{Z}_{23}, \tilde{Z}_{33}, \tilde{Z}_{43}, \tilde{Z}_{44}, \tilde{Z}_{53}, \tilde{Z}_{54}, \tilde{Z}_{63}$ and $\tilde{Z}_{64}$ are replaced by

$$
\begin{aligned}
& Z_{14}=\tilde{H}_{3-} \tilde{H}_{1-} ; Z_{23}=\tilde{H}_{2-} \tilde{r}_{-} ; Z_{33}=-2 m \pi \chi \tilde{H}_{1-} \tilde{r}_{-} ; Z_{43}=\tilde{S}_{r_{-}} ; Z_{44}=\tilde{C}_{r_{-}} \\
& Z_{53}=\tilde{H}_{1-} \tilde{S}_{r_{-}} ; Z_{54}=\tilde{H}_{1-} \tilde{C}_{r_{-}} ; Z_{63}=\tilde{H}_{1-} \tilde{r}_{-} \tilde{C}_{r_{-}} ; Z_{64}=-\tilde{H}_{1-} \tilde{r}_{-} \tilde{S}_{r_{-}}
\end{aligned}
$$

- $\lambda_{-}^{*}<\lambda ; \lambda_{+}^{*}<\lambda$ then $r_{-}^{2}<0 ; r_{+}^{2}<0$ and matrix $Z$ is given by the coefficients $\tilde{Z}_{i j}$ with $\tilde{Z}_{24}, \tilde{Z}_{34}, \tilde{Z}_{43}$, $\tilde{Z}_{44}, \tilde{Z}_{53}, \tilde{Z}_{54}, \tilde{Z}_{63}$ and $\tilde{Z}_{64}$ given in the previous equations and the other coefficients given by

$$
\begin{aligned}
& \tilde{Z}_{16}=Z_{16} ; \tilde{Z}_{25}=Z_{25} ; \tilde{Z}_{35}=Z_{35} ; \tilde{Z}_{55}=Z_{55} ; \tilde{Z}_{56}=Z_{56} ; \tilde{Z}_{65}=Z_{65} ; \tilde{Z}_{66}=Z_{66} \\
& Z_{12}=\tilde{H}_{3+} \tilde{H}_{1+} ; Z_{21}=\tilde{H}_{2+} \tilde{r}_{+} ; Z_{31}=-2 m \pi \chi \tilde{H}_{1+} \tilde{r}_{+} ; Z_{41}=\tilde{S}_{r_{+}} ; Z_{42}=\tilde{C}_{r_{+}} ; \\
& Z_{51}=\tilde{H}_{1+} \tilde{S}_{r_{+}} ; Z_{52}=\tilde{H}_{1+} \tilde{C}_{r_{+}} ; Z_{61}=\tilde{H}_{1+} \tilde{r}_{+} \tilde{C}_{r_{+}} ; Z_{62}=-\tilde{H}_{1+} \tilde{r}_{+} \tilde{S}_{r_{+}} \\
& Z_{11}=Z_{13}=Z_{15}=Z_{22}=Z_{24}=Z_{26}=Z_{32}=Z_{34}=Z_{36}=Z_{45}=Z_{46}=0 .
\end{aligned}
$$

Moreover, for a thin plate, the frequencies are obtained through the following characteristic equation [19], for $m \pi<\lambda$

$$
\begin{aligned}
& \lambda_{1} \tilde{\lambda}_{2}\left[\lambda^{2}-(1-v)^{2}(m \pi)^{4}\right]+(m \pi)^{2}\left[(1-2 v) \lambda^{2}-(1-v)^{2}(m \pi)^{4}\right] \sinh \frac{\lambda_{1}}{\chi} \sin \frac{\tilde{\lambda}_{2}}{\chi} . \\
& +\lambda_{1} \tilde{\lambda}_{2}\left[\lambda^{2}+(1-v)^{2}(m \pi)^{4}\right] \cosh \frac{\lambda_{1}}{\chi} \cos \frac{\tilde{\lambda}_{2}}{\chi}=0 .
\end{aligned}
$$

For $\lambda \leq m \pi, \tilde{\lambda}_{2}, \sin \left(\tilde{\lambda}_{2} / \chi\right)$, and $\cos \left(\tilde{\lambda}_{2} / \chi\right)$ are replaced by $\lambda_{2}, \sin \left(\lambda_{2} / \chi\right)$, and $\cos \left(\lambda_{2} / \chi\right)$, respectively.

\section{Numerical results: discussion}

Numerical results for the non-dimensional frequency $\lambda$ were obtained for each of the six cases considered, an aspect ratio equal to 1 (square plate) and 2 and a Poisson ratio 0.3 , following the closed-form solution obtained through the Navier's approach for an all edges simply supported plate (Table 2) and the exact solutions for the other cases given by the Lévy approach (see Tables 3, 4, 5).

For each case previously presented (SCSC, SFSF, SSSC, SSSF, SCSF), the first natural frequencies have been compared with those of the classical plate theory in the monograph of Leissa [19], those of Wang and Wang [36] and those obtained by Hashemi [26] (see Table 5). Different shear correction factors $\kappa^{2}$ are used in the literature. Hashemi and Arsanjani [26] or Wang and Wang [36] took 8.667. Hereinafter, this value is retained in order to compare our results with the results of the literature. However, other values could be used: $\kappa^{2}=\pi^{2} / 12$ (used by Mindlin [5,35]), $\kappa^{2}=0.822, \kappa^{2}=0.88$ [37,38]. Three different thickness ratios are considered: $\bar{h}=0.01 ; \bar{h}=0.1$ and $\bar{h}=0.2$. In each table, the five lowest frequencies are displayed.

All the results presented in this paper are obtained from the exact solution and so are extremely accurate, much more than any numerical method such as the Rayleigh-Ritz method that includes naturally some approximations. Thus, considering the original Uflyand-Mindlin theory, the results match with those obtained by Hashemi and Arsanjani for the original Uflyand-Mindlin plate theory. An error smaller than $1 \%$ can occur due to the numerical calculations. The same calculations have been performed for $\kappa^{2}=0.822$ and coincide 


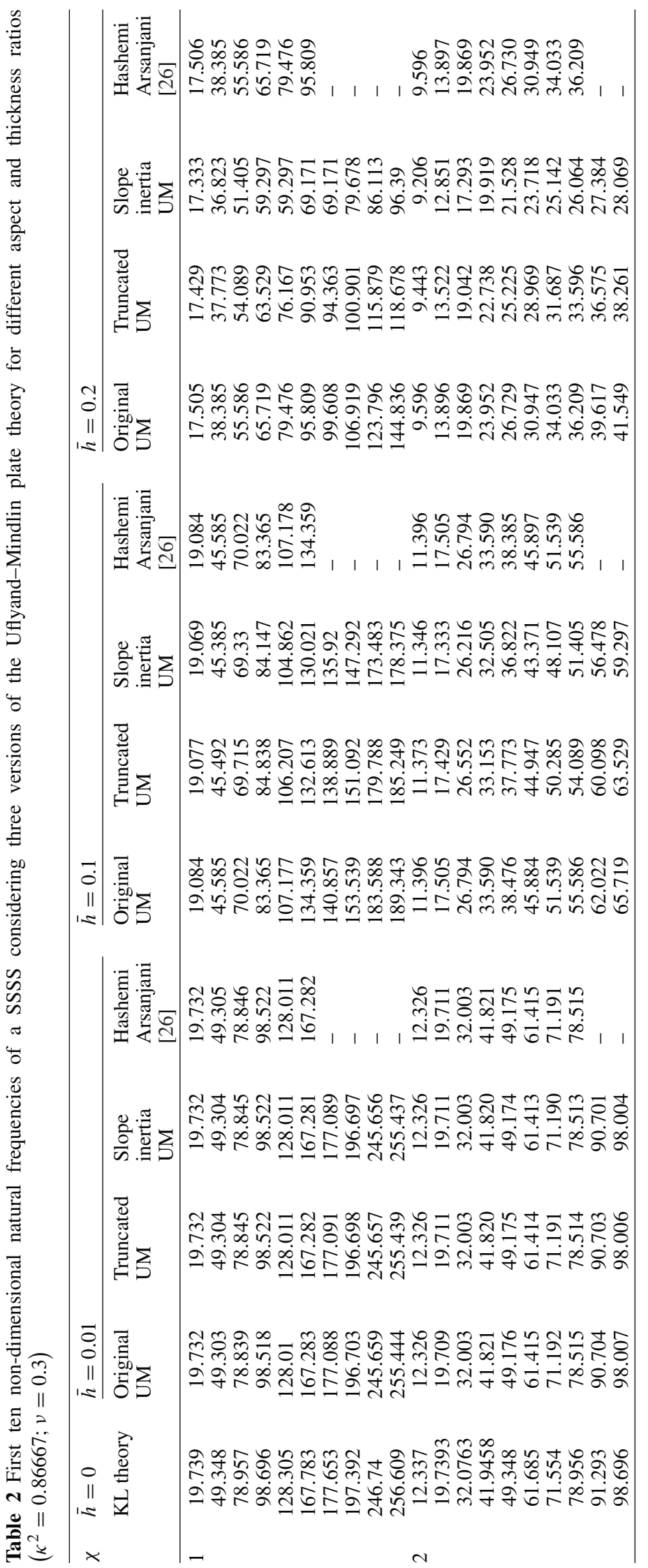




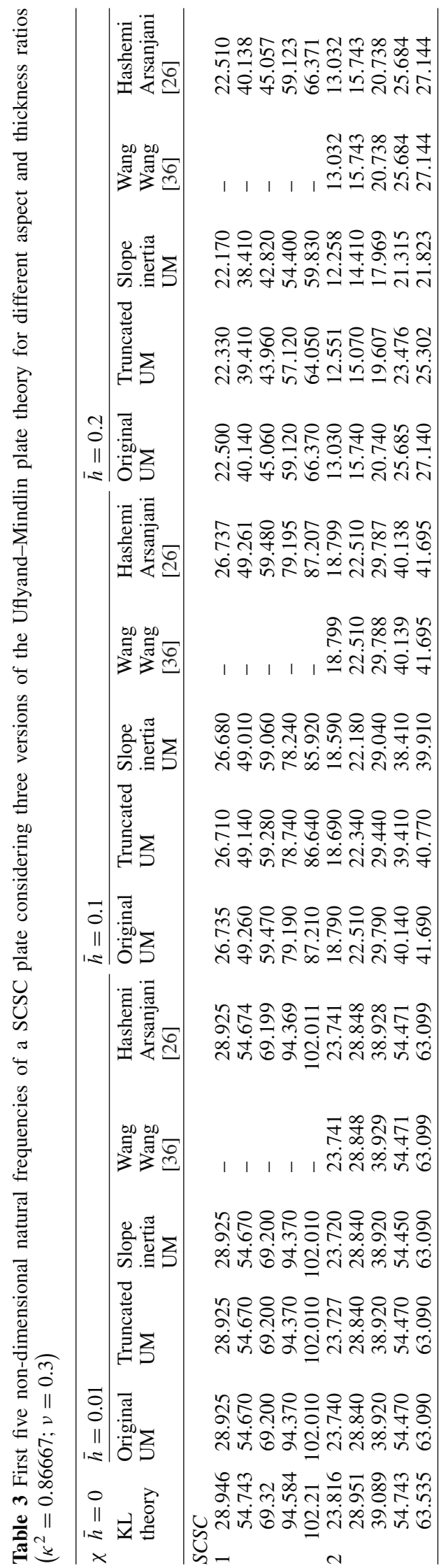




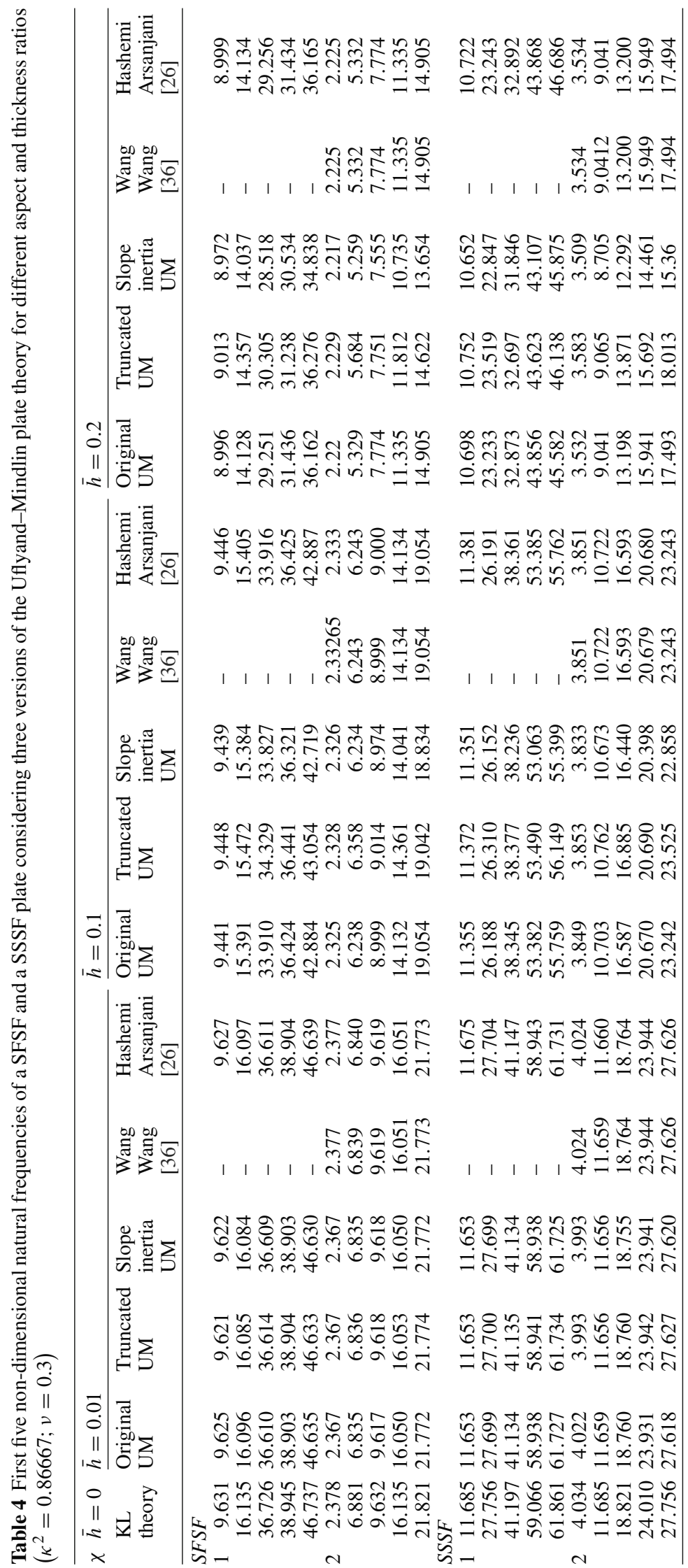




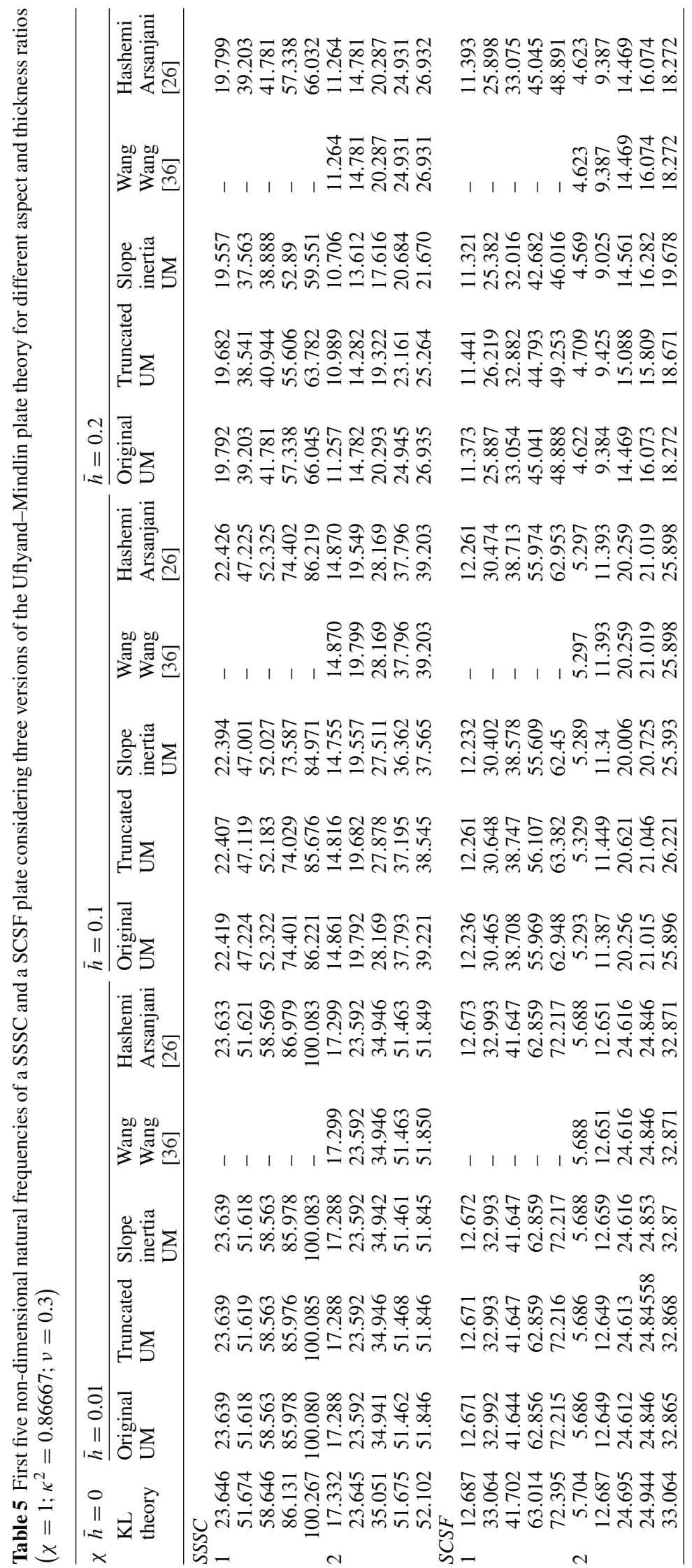


with those obtained by Dawe [38]. This validates the accuracy of the calculations and the solutions developed in this paper.

In their paper, Liew et al. [13] calculate the natural frequencies by using the numerical Rayleigh-Ritz method. This method is extremely fast to implement and can be used in any situation. However, this gives an upper bound, but our results and it allow to question the accuracy of such methods.

The objective of the present paper is to present the analytical determination of the natural frequencies of thick plates considering three versions of the Uflyand-Mindlin theory by using the Navier approach (all edges simply supported) or, more generally, the Lévy method (at least two opposite edges simply supported). Whatever the boundary conditions, the thickness, or the aspect ratio, the three models provide extremely close results. The natural frequencies are bigger for the truncated Uflyand-Mindlin theory than for the two other models, whereas the Uflyand-Mindlin theory based on slope inertia provides lower frequencies. The analytical difference between the truncated Uflyand-Mindlin theory and the Uflyand-Mindlin theory based on slope inertia is clearly established in the case of a plate with all edges simply supported (see Eq. (47))

Considering a square plate $(\alpha=1)$, the modes $(1,2)$ and $(2,1)$ provide the same natural frequency in the case of SSSS, SCSC, and SFSF due to the symmetries. This result confirms the determination of the analytical solutions in which the geometrical symmetries are used to simplify the calculations.

As explained above, according to Eq. (48), for any case considered in this paper, the wave forms are, of course, sine functions in the $x$ direction. When the two other edges are both clamped or free, the node lines will be also parallel to the $x$ axis. For the other cases the forms are not straight and only approximately sinusoidal.

The influence of thickness ratio on the non-dimensional natural frequency considering different boundary conditions is established by taking the aspect ratio $\alpha$ constant and varying $\bar{h}$ from 0.01 to 0.2 . For a thickness ratio equal to 0.01 , the results are close to those obtained from the thin plate theory (classical plate theory). When the thickness ratio is small, the rotary inertia and the shear effect should have no influence. Thus, all the results (equations and solutions) for a thin plate, developed by Leissa [19], are obtainable by taking $\kappa^{2}$ going to infinity. It can be observed that, as the thickness ratio increases, the frequency parameter decreases with the thickness ratio increasing. Indeed, when $\bar{h}$ is large, the rotary inertia and the shear effect cannot be ignored. This influence becomes more important when the the mode of the frequency increases.

To study the effect of the boundary conditions, the thickness and aspect ratios are taken equal to 0.2 and 1 , respectively, and the five first non-dimensional frequencies are listed in Tables 2, 3, 4 and 5 for the six different boundary conditions. Irrespective of the model, the difference between the models increases with the order of the natural frequency. For higher orders, the Uflyand-Mindlin plate theory provides lower non-dimensional natural frequencies than the two other Uflyand-Mindlin models. This difference increases with the mode number. From this table, it is established that $\lambda_{S F S F}<\lambda_{S S S F}<\lambda_{S C S F}<\lambda_{S S S S}<\lambda_{\text {SSSC }}<\lambda_{\text {SCSC }}$. Thus, the lowest frequencies are obtained when one of the edges is free. In contrast to the SFSF, the SCSC provides the highest frequencies. For instance, considering the original Uflyand-Mindlin theory, the fundamental natural frequency is $60 \%$ more important for the SCSC case (22.509) than for the SFSF case (8.997). Thus, higher constraints at the edges increase the overall rigidity of the plate, resulting in a higher frequency response as expected [26]. It is worth noting that there is no apparent correlation between the boundary conditions and the difference between the Uflyand-Mindlin theories and the Kirchhoff-Love model.

Furthermore, the comparison of the non-dimensional natural frequency for an aspect ratio equal to 1 (square plate) and 2 shows that it decreases with increasing plate aspect ratio if the relative thickness ratio and boundary conditions are kept constant.

Ultimately, it is worth noting that for the original Uflyand-Mindlin plate theory, two cases have to be considered, following the values of the natural frequency. Thus, the truncated Uflyand-Mindlin plate theory and the Uflyand-Mindlin plate theory based on slope inertia provide very accurate results and are much simpler compared to the original one.

\section{Conclusion}

The aim of this study was to analyze the different models describing the mechanical behavior of a plate under free vibrations. Three models have been studied: original Uflyand-Mindlin, truncated, and Uflyand-Mindlin based on slope inertia. For each of these models, the first five natural frequencies of a plate have been calculated, considering six different boundary conditions. The difference between the models depends on the aspect ratio, the order of the frequency, and the boundary conditions. When the flexural rigidity of the plate increases (for instance, by considering one of the edges free), the natural frequencies decrease, whereas the differences between the models increase. 
Acknowledgements The authors would like to thank the editor of Acta Mechanica, Professor Hans Irschik, and the anonymous reviewers who have greatly contributed to improve the exposition of the paper.

\section{References}

1. Kirchhoff, G.: Über das Gleichgewicht und die Bewegung einer elastischen Scheibe. J. Angew. Math. 40, 51-88 (1850) (in German)

2. Lee, K.H., Lim, G.T., Wang, C.M.: Thick Lévy plates re-visited. Int. J. Solids Struct. 39, 127-144 (2002)

3. Reissner, E.: The effect of transverse shear deformation on the bending of elastic plates. J., Appl. Mech. 12, A69-A77 (1946)

4. Uflyand, Y.S.: The propagation of waves in the transverse vibrations of bars and plates. Akad. Nauk SSSR Prikl. Math. Mech. 12, 287-461 (1948)

5. Mindlin, R.D.: Influence of rotatory inertia and shear on flexural motions of isotropic, elastic plates. Trans. ASME J. Appl. Mech. 18, 31-38 (1951)

6. Elishakoff, I.: Generalization of the Bolotin's dynamic edge effect method for vibration analysis of Mindlin plates. In: Cuschieri, J.M., Glegg, S.A.L., Yeager, D.M., (eds). NOISE-CON 94. Proceedings of the 1994 National Conference on Noise Control Engineering; 1994 May 01-04; Fort Lauderdale

7. Elishakoff, I., Hache, F., Challamel, N.: Vibrations of asymptotically and variationally based Uflyand-Mindlin plate models. Int. J. Eng. Sci. 116, 58-73 (2017)

8. Irschik, H.: Membrane-type eigenmotions of mindlin plates. Acta Mech. 55, 1-20 (1985)

9. Irschik, H., Heuer, R., Ziegler, F.: Statics and dynamics of simply supported polygonal Reissner-Mindlin plates by analogy. Arch. Appl. Mech. 70, 231-244 (2000)

10. Brunelle, E.J., Roberts, S.R.: Initially stressed Mindlin plates. AIAA J. 12, 1036-1045 (1974)

11. Brunelle, E.J.: Buckling of transversely isotropic Mindlin plates. AIAA J. 9, 1018-1022 (1971)

12. Sharma, A., Sharda, H.B., Nath, Y.: Stability and Vibration of Mindlin sector plates: an analytical approach. AIAA J. 4, 1109-1116 (2005)

13. Liew, K.M., Wang, C.M., Xiang, Y., Kitipornchai, S.: Vibration of Mindlin plates: programming the p-Version Ritz method. Elsevier, Oxford, UK, Oxford (1998)

14. Zenkour, A.M.: Buckling and free vibration of elastic plates using simple and mixed shear deformation theories. Acta Mech. 146, 183-197 (2001)

15. Naumenko, K., Altenbach, J., Altenbach, H., Naumenko, V.K.: Closed and approximate analytical solutions for rectangular Mindlin plates. Acta Mech. 147, 153-172 (2001)

16. Eftekhari, S.A., Jafari, A.A.: A simple and accurate Ritz formulation for free vibration of thick rectangular and skew plates with general boundary conditions. Acta Mech. 224, 193-209 (2013)

17. Rayleigh, J.W.: Theory of Sound, vol. 1. Macmillan, London (1877)

18. Ritz, W.: Über eine neue Methode zur Lösung gewisser Variationsprobleme der mathematischen Physik. J. Angew. Math. 135, 1-61 (1909) (in German)

19. Leissa, A.W.: Vibration of Plates. U.S Government Printing Office, NASA SP-160, reprinted by the Acoustical Society of America (1969)

20. Bhat, R.B.: Natural frequencies of rectangular plates using characteristic orthogonal polynomials in the Rayleigh-Ritz method. J. Sound Vib. 102, 493-499 (1985)

21. Bhat, R.B.: Flexural vibration of polygonal plates using characteristics orthogonal polynomials in two-variables. J. Sound Vib. 114, 65-71 (1987)

22. Liew, K.M., Lam, K.Y.: Application of two-dimensional orthogonal plate function to flexural vibration of skew plates. J. Sound Vib. 139, 241-252 (1990)

23. Leissa, A.W.: The free vibration of rectangular plates. J. Sound Vib. 31, 257-293 (1973)

24. Gorman, D.J.: Free Vibration Analysis of Rectangular Plates. Elsevier-North Holland Publishing Co, New York (1982)

25. Gorman, D.J., Ding, W.: Accurate free vibration analysis of point supported Mindlin plates by the superposition method. J. Sound Vib. 219, 265-277 (1999)

26. Hashemi, S.H., Arsanjani, M.: Exact characteristic equations for some of classical boundary conditions of vibrating moderately thick plates. Int. J. Solids Struct. 45, 819-853 (2005)

27. Hashemi, S.H., Khorshidi, K., Amabili, M.: Exact solution for linear buckling of rectangular Mindlin plates. J. Sound Vib. 315, 318-342 (2008)

28. Mindlin, R.D.: In: Yang, J. (ed.) An Introduction to the Mathematical Theory of Vibrations of Elastic Plates. World Scientific Publishing Co, Singapore (2006) (based on the monograph written by R.D. Mindlin in 1955)

29. Navier, C.L.M.H.: Extrait des recherches sur la flexion des plans élastiques. Bull. Sci. Soc. Philomarhiques de Paris 5, 95-102 (1823)

30. Chen, W.C., Liu, W.H.: Deflections and free vibration of laminated plates-Lévy-type solutions. Int. J. Mech. Sci. 32, 779-793 (1990)

31. Szilard, R.: Theory and Analysis of Plates. Prentice-Hall, Englewood Cliffs, New Jersey (1974)

32. Jomehzadeh, E., Saidi, A.R.: A Lévy type solution for free vibration analysis of a nano-plate considering the small scale effect. Recent Adv. Vib. Anal. 47-58 (2011)

33. Wang, C.M., Lim, G.T., Reddy, J.N., Lee, K.H.: Relationships between bending solutions of Reissner and Mindlin plate theories. Eng. Struct. 23, 838-849 (2001)

34. Wang, C.M.: Natural frequencies formula for simply supported Mindlin plates. J. Vib. Acoust. 116, 536-540 (1994)

35. Mindlin, R.D., Schacknow, A., Deresiewicz, H.: Flexural vibrations of rectangular plates. Trans. ASME J. Appl. Mech. 23, 430-436 (1956) 
36. Wang, C.Y., Wang, C.M.: Structural Vibration: Exact Solutions for Strings, Membranes, Beams, and Plates. CRC Press, Boca Raton (2014)

37. Srinivas, S.R., Joga Rao, C.V., Rao, A.K.: An exact analysis for vibration of simply supported homogeneous and laminated thick rectangular plates. J. Sound Vib. 12, 187-199 (1970)

38. Dawe, D.J.: Finite strip models for vibration of Mindlin plates. J. Sound Vib. 59, 441-452 (1978) 